\title{
Small-Scale Structure in the Lithosphere and Asthenosphere Deduced From Arrival Time and Amplitude Fluctuations at NORSAR
}

\author{
STANLEY M. FlatTÉ AND RU-SHAN WU \\ Physics Department and Institute of Tectonics, University of California, Santa Cruz
}

\begin{abstract}
We analyze the pattem of phase and amplitude variations of seismic waves across the NORSAR array on a statistical basis in order to determine the statistical distribution of heterogeneities under NORSAR. Important observables that have been analyzed in the past are the phase (or travel time) and log amplitude variances and the transverse coherence functions (TCFs) of phase and amplitude fluctuations. We propose and develop the theory and methods of using other observables to reduce the degree of nouniqueness and increase the spatial resolution of the analysis. Most important are the angular coherence functions (ACFs), which characterize quantitatively the change in the pattem of fluctuations across the array from one incoming angle (or beam) to another and which have a different sensitivity to the depth distribution of heterogeneities than the TCFs. A combination of the ACFs and TCFs allows estimation of the power spectra of the $P$ wave speed variations under the array as a function of depth. We use data for phase fluctuations from 104 incident beams and amplitude fluctuations from 185 beams with $2-\mathrm{Hz}$ center frequency at NORSAR to calculate the three ACFs and three TCFs (of phase, log amplitude, and their cross coherence). The measured $\mathrm{ms}$ travel time fluctuation is $0.135 \mathrm{~s}$, and the $\mathrm{ms} \log$ amplitude fluctuation is 0.41 . The halfcoherence widths of the ACFs are $3^{\circ}$ for $\log$ amplitude and $9^{\circ}$ for phase. The half-coherence widths of the TCFs are $18 \mathrm{~km}$ for phase and less than the minimum separation between the elements of the array for log amplitude. In order to account for these features of the data, we adopt a two-overlapping-layer model for lithospheric and asthenospheric heterogeneities underneath NORSAR, with spectra that are band-limited between the wavelengths of 5.5 and $110 \mathrm{~km}$. Our best model has an upper layer with a flat power spectrum extending from the surface to about $200 \mathrm{~km}$, and a lower layer with a $K^{-4}$ power spectrum extending from 15 to $250 \mathrm{~km}$. The latter spectrum corresponds to an exponential correlation function with scale larger than the observation aperture $(110 \mathrm{~km})$. The $\mathrm{ms} P$ wave speed variations lie in the range $1-4 \%$. The small scale heterogeneities may be attributed to clustered cracks or intrusions; the larger-scale wavespeed heterogeneities are temperature or compositional heterogeneities that may be related to chemical differentiation, or dynamical processes in the boundary layer of mantle convection.
\end{abstract}

\section{INTRODUCTION}

Observations of direct $P$ wave amplitude and arrival time fluctuations [Aki, 1973; Capon, 1974; Capon and Berteussen, 1974; Berteussen et al., 1975a, b; Powell and Meltzer, 1984], coda strength [Aki, 1981; Sato, 1982; Wu and Aki, 1985a, b], and attenuation by scattering [Aki and Chouet, 1975; Aki, 1980; $W u, 1982 ;$ Sato, 1982] have all been used in attempts to determine some statistical characteristics of small-scale structure in the Earth. Statistical analyses of amplitude and arrival time fluctuations have previously involved the variances of log amplitude and arrival time, the covariance of log amplitude and arrival time, and the coherence functions of $\log$ amplitude and arrival time as functions of spatial separation along the Earth's surface (the transverse coherence functions, or TCFs) [Aki, 1973; Capon, 1974; Capon and Berteussen, 1974; Berteussen et $a l ., 1975 a, b$; Powell and Meltzer, 1984].

Theoretical analyses of the observations assume a particular medium structure and, by various techniques, compare theoretical predictions of seismic wave properties to the observations. Previous seismic wave analyses followed Chernov [1960], who used the Rytov and Fresnel approximations to connect wave fluctuations to medium variations. His expressions involving general random media in the space domain are formidable, but he evaluated them explicitly for a statistically uniform and iso-

Copyright 1988 by the American Geophysical Union.

Paper number 7B5081.

0148-0227/88/007B-5081\$05.00 tropic random medium with a Gaussian correlation function; his resulting expressions have been used by previous workers to obtain strengths and scale sizes of the $P$ wave speed variations in the lithosphere by modeling it as a uniform layer of a singlescale random medium.

This paper has several purposes: first, to point out that the log amplitude and arrival time fluctuations at large seismic arrays like NORSAR and LASA have further important statistical information that has not been utilized in previous analyses, especially the coherence function of waves from different sources, and hence different incoming directions; second, to present a statistical analysis of NORSAR data that includes this new information; third, to present results from the modem theory of wave propagation through random media (WPRM) that are based on the parabolic wave equation approach and are somewhat simpler than Chernov's because they are formulated partially in the spectral domain and can accommodate easily any model of the medium spectrum; and fourth, to present a model of the inhomogeneities in the lithosphere and asthenosphere under the NORSAR array that is consistent with the available data.

Before discussing the most interesting of the new information available, it is important to point out that there are three variances involved in arrival time and log amplitude fluctuations at a given seismic frequency; the variances of arrival time and $\log$ amplitude, and the covariance between the two. Each of these variances leads to a coherence function of any variable being investigated; for example, previous analyses [Aki, 1973; Capon, 1974; Capon and Berteussen, 1974; Berteussen et al., 1975a, b; 
Powell and Meltzer, 1984] have used the arrival time and log amplitude transverse coherence functions, but we will be analyzing here for the first time the transverse cross-coherence function between arrival time and log amplitude.

The main new information that can be obtained from an array that detects waves arriving from many directions ("beams") is the set of three angular coherence functions (ACFs): that is, the coherence functions of arrival time, log amplitude, and their cross coherence, all as a function of the angle between two incoming directions. Since the difference in direction between two beams may be as small as $1^{\circ}$, the information at a given receiver probes (in a statistical sense) inhomogeneities that are quite small: of the order of $1 \mathrm{~km}$ at $60-\mathrm{km}$ depth. The transverse coherence functions from a coarse array with $-10-\mathrm{km}$ spacing cannot probe to scales smaller than $10 \mathrm{~km}$.

Data on arrival time and amplitude fluctuations of the firstarrival teleseismic $P$ wave signal are analyzed in a different fashion than data involving large-angle scattering of wave energy. The difference involves the realization that the full wave equation need not be solved, but rather a simpler equation: the parabolic wave equation (PWE) that adequately treats waves in a narrow angular cone. (Note that if coda is interpreted as large-angle scattered waves, it cannot be treated in the same way.) One consequence of using the PWE is that the theoretical formulas are easily expressed in terms of an integral along the unperturbed ray, rather than as a volume integral over all of space. Section 2 briefly introduces the modern theory of WPRM theory based on the PWE and "weak" fluctuations. Section 3 specifically describes the theory of the angular coherence functions.

The NORSAR data used in our analysis consist of the travel time anomalies [Berteussen, 1974] at 22 subarrays for 104 beams; and the log amplitude fluctuations [Berteussen and Husebye, 1974] at 22 subarrays for 185 beams. All the beams have incoming directions within a $35^{\circ}$ cone around the zenith. The smallest distance between subarrays is about $10 \mathrm{~km}$, and the largest is about $110 \mathrm{~km}$. The data used were filtered for $1-3 \mathrm{~Hz}$, so the nominal frequency is $2 \mathrm{~Hz}$. It is important to realize that the data that we analyze are insensitive to inhomogeneities with wavelengths greater than about $100 \mathrm{~km}$ because of the finite size of the array and are also insensitive to inhomogeneity wavelengths less than about $5 \mathrm{~km}$ because of two effects: first the data are averages over subarrays that are $7 \mathrm{~km}$ in diameter, and second, the wavelengths of the seismic waves are about 4 $\mathrm{km}$. Section 4 describes the data analysis and presents results for coherence functions at NORSAR.

It does not take a sophisticated theory to draw some dramatic conclusions from this new analysis of NORSAR data. Briefly, the $\log$ amplitude ACF drops rapidly, reaching a value of 0.5 at an angle of $2^{\circ}$, followed by a more gradual drop to 0.1 at $10^{\circ}$. In contrast, the arrival-time ACF drops much more slowly, implying much larger-scale structure. Yet all the TCFs at first glance have scales in the range of 10-20 km. Section 5 contains the quantitative comparison of weak scattering models with the NORSAR data. We have found that medium spectral models that are homogeneous in depth (that is, the spectrum does not change with depth) down to a cutoff depth between 0 and 500 $\mathrm{km}$ cannot fit all the data at once; in particular, such models, which can fit the TCFs by themselves, cannot simultaneously fit the shapes of the ACFs. We suggest a two-overlapping-layer model in which the medium spectrum is characterized by a power law $K^{-p}$ over the sensitive wave number band $(5<\lambda<100 \mathrm{~km})$. An upper layer with significant small-scale structure $(p=0)$ spans $0<z<200 \mathrm{~km}$ and a lower layer with strength concentrated at large scales $(p=4)$ spans $15<z<250 \mathrm{~km}$. The $P$ wave $\mathrm{ms}$ velocity variations in our model vary between 1 and $4 \%$. A specific prediction of the model that we suggest is a rapid drop in the log amplitude TCF for separations of a few kilometers, followed by a more gradual drop with a scale of tens of kilometers. We have checked this prediction by determining the TCF of $\log$ amplitude from the individual stations at NORSAR, using 13 nuclear explosion events. We find consistency with our prediction, giving us some confidence in our interpretation.

\section{THEORY OF WPRM}

Many aspects of seismic wave propagation in the Earth involve examples of a more general branch of science; namely, wave propagation through random media (WPRM). Progress in this field has involved coupling of the theory of WPRM with investigation into the detailed character of the (generally fluid) random medium. At least two theoretical approaches may be taken: first, the problem may be approached from a deterministic point of view in which the analysis is carried out for particular complex media. Numerical simulation is the extreme of this point of view. Second, the problem may be treated statistically from the outset. In this approach, one assumes a spectral model for the medium and attempts by analytical means to predict the statistical behavior of the propagating wave field. Here we take this second point of view.

The modern theory of WPRM may be said to have begun in the late 1940s and early 1950s [Bergmann, 1946; Mintzer, 1953; Chernov, 1960; Tatarskii, 1971] when researchers used perturbation techniques to develop general formulas for propagation through weak fluctuations. These formulas involved the Bom approximation and hence a volume integral over the medium inhomogeneities. In comparing with experiment they assumed that the medium was characterized by a Gaussian correlation function, but unfortunately, no natural medium is known with this property.

The problem of radio wave propagation in and through the Earth's ionosphere has been of interest since the 1940s. This field made a crucial contribution to WPRM through the work of Leontovich and Fock [1946] and Fock [1950], who introduced the parabolic equation method, which treats waves that are concentrated within a small angular region around the direction of propagation. Nearly all subsequent analytic work has used the parabolic equation as a starting point, which restricts validity to waves with directions confined within a cone of full angle about $30^{\circ}$. A great advantage of the parabolic equation is that it results in formulas requiring line integrals along deterministic rays, rather than volume integrals.

A major step forward in WPRM was taken in the 1960s, in response to developing understanding of Kolmogorov's pioneering characterization of the $p=11 / 3$ power law spectrum of homogeneous, isotropic turbulence (HIT). A theory for wave propagation through HIT was developed, primarily by Soviet workers, and successfully applied in the case of weak fluctuations to light transmission through the atmosphere, making use of the parabolic equation. Their work was summarized in the influential book by Tatarskii [1971] and a review article by Prokhorov et al. [1975], and more recent results in this field are covered in the review articles by Ishimaru [1977], Fante [1980], 
and Tatarskii and Zavorotnyi [1980], as well as in the book edited by Strohbehn [1978].

It is now understood that weak fluctuation theory applies when the variance of $\log$ amplitude is small compared with unity. We realize that many seismic data sets have large intensity variations, but in this paper we consider only those for which the log amplitude variance is small. For example, the NORSAR data set of subarray amplitude variations has a $\log$ amplitude variance of 0.2 . We defer discussion of the problem of strong fluctuations to the future.

Seismic rays often tum through large angles, so that the parabolic approximation is not clearly appropriate. However, if the wave fluctuations are caused by medium variations that are confined to regions near the source and receiver (within a few hundred kilometers), then the rays are not changing their angles significantly, and the parabolic approximation is valid.

Work on fluctuations in sound transmission through the ocean during the 1960s was largely a misguided attempt to graft the concept of HIT onto ocean variability [Tatarskii, 1971]. By the mid-1970s, oceanographers had identified internal waves as the most important source of variability on time scales from a few minutes to a day [Garrett and Munk, 1975].

The ocean internal wave medium provided a challenge to those interested in WPRM, particularly in strong fluctuations. A significant response to this challenge was developed over the late 1970s. The first success in this area was achieved for weak fluctuations by Munk and Zachariasen [1976], whose absolute calculations of variances in phase and log amplitude from internal wave effects were within a factor of 2 of the available experimental results. Strong fluctuations were another matter.

A further basic step forward took place between 1975 and 1977 , with the consideration of the arbitrary field of wave speed in Feynman's path integral as a statistical random medium [Dashen, 1979; Flatté et al., 1979], in combination with the understanding of the ocean internal wave field as the source of medium fluctuations. These ideas led to successful comparisons with a number of ocean acoustics experiments, summarized in the monograph by Flatté et al. [1979], the review article by Flatte [1983], and subsequent publications [Dashen et al., 1985; Reynolds et al., 1985; Flatté and Stoughton, 1986; Stoughton et al., 1986; Flatté, et al., 1987a, b].

With this background in mind, we now apply parabolic equation, weak-scattering theory to $P$ wave teleseismic propagation. We take the point of view that the seismic waves arrive a few hundred kilometers below an array like NORSAR in essentially plane wave form: that any effect due to inhomogeneities near the source is too large scale to be observed by a $100-\mathrm{km}$ array and that the very deep mantle has imposed no significant structure on the wave that has scales less than the $100-\mathrm{km}$ size of the array. The reason that the inhomogeneities near the source have little effect is that only a very small range of initial angles at the source is seen by the array, and the spreading of the ray tube magnifies any small-scale fluctuations of the wave front into large-scale fluctuations at the receiver.

Furthermore we assume that the effect on the arrival time and amplitude fluctuations of inhomogeneities within a few hundred kilometers depth below the array results mainly from wave scattering within a reasonably narrow cone. We realize that coda can represent scattering at larger angles; however, the first few seconds of $P$ wave arrivals are restricted to small angles.

Since NORSAR data (by subarray) show a log amplitude variance of 0.2 , we use weak fluctuation theory. It has been

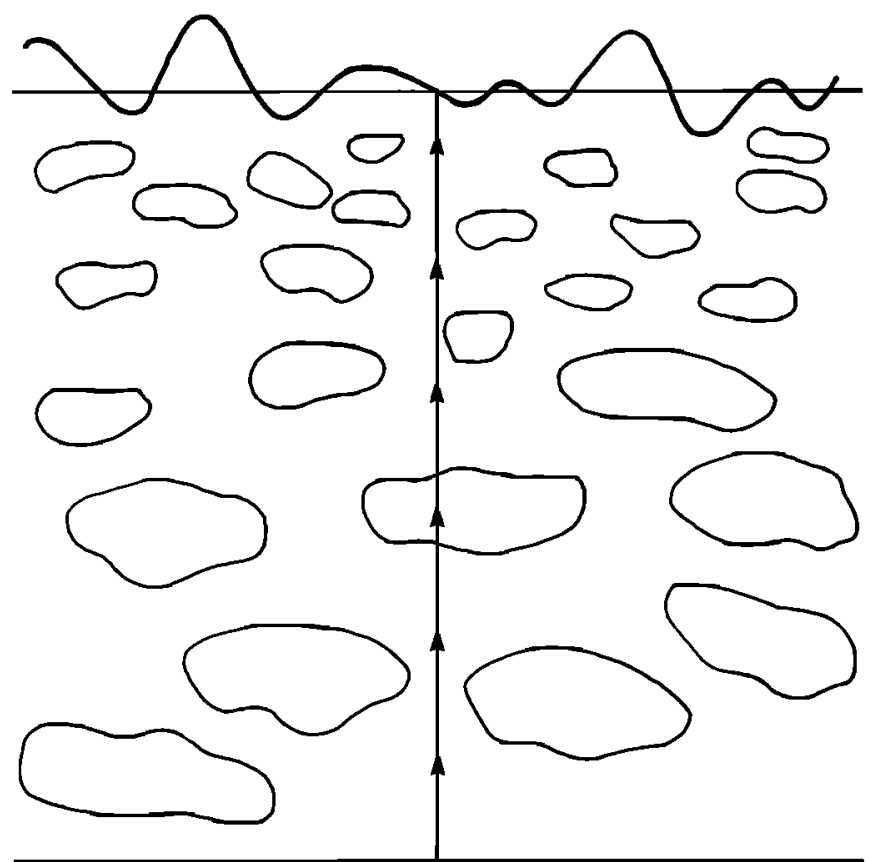

Fig. 1. Schematic of $P$ wave propagation through the upper mantle, lithosphere, and crust to a surface seismic array. The heavy solid straight line is the incident wave front, the light solid horizontal line is the wave front arriving at the receiving array in the absence of fluctuations. The wavy line could represent either the wave front arrival time accros the array or the intensity at each point in the arriving wave front.

established that even if intensity fluctuations are large, if they are averaged over a small source (or receiver) region, such that the residual (larger scale) intensity fluctuations are small, then weak-fluctuation theory can be applied [Wang et al., 1978]. There are probably aspects of this averaging in the NORSAR data because of the $7-\mathrm{km}$ width of a typical subarray and because of finite wavelength effects, which result in the observed log amplitude variance of 0.2 .

Finally, we neglect the small deterministic refraction in the few hundred kilometers below the array; in the absence of inhomogeneities, the wave fronts remain plane in the same direction. (See Figure 1.)

We now state the results of weak-scattering theory under the above restrictions. First, define the medium $P$ wave speed as a function of position:

$$
C(\underline{x})=C_{0}[1+\mu(\underline{x})]
$$

where $C_{o}$ is a reference deterministic speed that might depend on depth $z$, and $\mu(\underline{x})$ is a random function with zero mean that represents inhomogeneities. The statistics of $\mu$ are assumed to be quasi-homogeneous. That is, we can define a spectrum at depth $z$, and this spectrum is allowed to change slowly with depth. In this case, the character of $\mu(\underline{x})$ is described by a three-dimensional spectrum $W(\underline{K}, z)$, such that

$$
\left\langle\mu(\underline{x}) \mu\left(\underline{x}^{\prime}\right)\right\rangle=\int d^{3} \underline{K} W(\underline{K}, z) e^{i K \cdot\left(x-x^{\prime}\right)}
$$

where we have assumed that the spectrum depends on a threedimensional wave vector $\underset{K}{K}$ and depth $z$, and the angle brackets represent an average over the statistical ensemble of random media. Since the spectrum does not depend on transverse posi- 
tion and depends only slowly on depth $z$, the medium correlation function $\left\langle\mu(x) \mu\left(x^{\prime}\right)\right\rangle$ depends only on the difference vector $x-x^{\prime}$, except for a slow dependence on $z$ which is implicit. Note the normalization of the spectrum is such that

$$
<\mu^{2}>=\int d^{3} \underline{K} W(\underline{K}, z)
$$

where $\left\langle\mu^{2}\right\rangle$ is the variance of the fractional change in wave speed due to the random inhomogeneities; it can depend on depth $z$.

Consider a given frequency $\omega$ in the incoming wave. That frequency has wave number $k=\omega / C_{0}$. Let the complex wave function arriving at the center of the array be

$$
\Psi \equiv A e^{i \phi} \Psi_{0}
$$

where $\Psi_{0}$ is the wave function that would arrive in the absence of the random inhomogeneities and where the amplitude $A$ and phase $\phi$ are defined in this relation. Define the log amplitude as $u$, so that

$$
\ln \left(\Psi / \Psi_{0}\right)=u+i \phi
$$

It is known in the case of weak scattering confined to an narrow cone, in an intrinsically dispersion-free medium, that the arrival time fluctuation $\tau$ and the phase fluctuation $\phi$ are in one-to-one correspondence:

$$
\phi=\omega \pi
$$

We now point out that for a specific realization of inhomogeneities, $u$ and $\phi$ are functions of both the receiver position $\underline{x}$ and the source position, represented by a unit vector $\theta$ describing the direction of the incoming plane wave. (See Figure 1.)

Note first that we have made the approximation that the random medium is "horizontally homogeneous"; that is, the spectrum is independent of horizontal position. We also consider waves arriving within $30^{\circ}$ of the zenith, so we avoid some simple geometrical corrections that are important only for highly slanted rays. We can split the three-dimensional wave number

$K$ into a component along the $z$ direction $K_{z}$ and a twodimensional vector $K_{T}$ transverse to z: that is, $K_{T}$ is in the $x-y$ plane. Then the medium spectrum can be expressed as

$$
W(\underline{K}, z) \equiv W\left(K_{z}, \underline{K}_{T}, z\right)
$$

With the above definitions and approximations, the result [Munk and Zachariasen, 1976; Flatté et al., 1979; S.M. Flatté and T. Moody, manuscript in preparation, 1987] for the variances of the seismic wave fluctuations at the surface of the earth are

$$
\begin{gathered}
<u^{2}>=2 \pi k^{2} \int_{0}^{R} d z \int d^{2} \underline{K}_{T} W\left(0, K_{T}, z\right) \sin ^{2}\left[K_{T}^{2} z / 2 k\right] \\
<\phi^{2}>=2 \pi k^{2} \int_{0}^{R} d z d^{2} \underline{K}_{T} W\left(0, K_{T}, z\right) \cos ^{2}\left[K_{T}^{2} z / 2 k\right] \\
<u \phi>=2 \pi k^{2} \int_{0}^{R} d z \int d^{2} \underline{K}_{T} W\left(0, K_{T}, z\right) \\
\sin \left[K_{T}{ }^{2} z / 2 k\right] \cos \left[K_{T}{ }^{2} z / 2 k\right]
\end{gathered}
$$

where $R$ is the depth of the deepest inhomogeneities being analyzed.

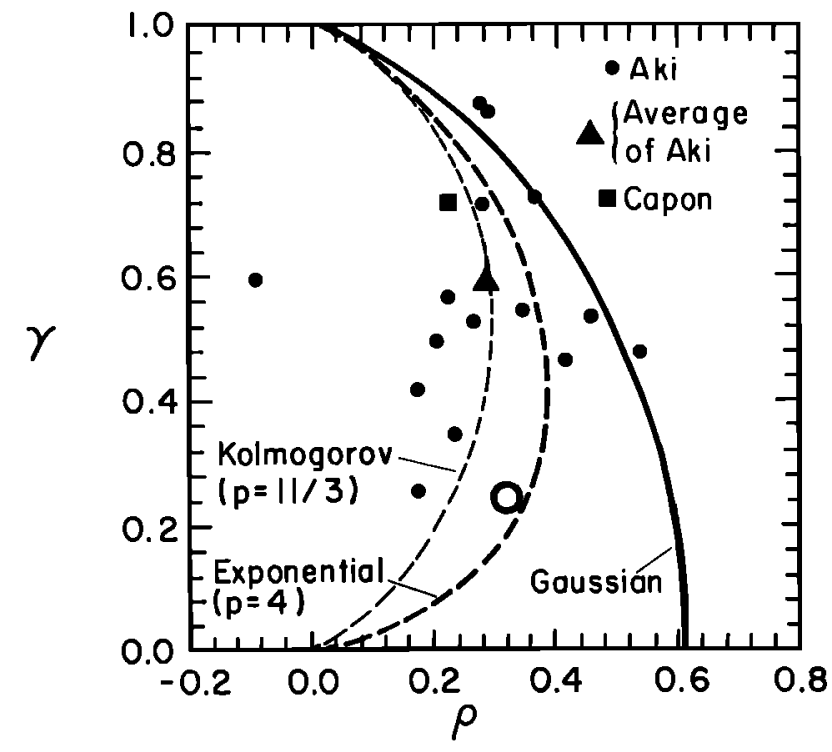

Fig. 2. Variance ratio $\gamma$ versus amplitude-phase correlation $p$ for uniform extended media. The solid circles are from Aki [1973]. The solid squares are from Capon [1974]. The open circle comes from our analysis of NORSAR data. The quantity $p$ is the spectral power law index (equation (30)). Position along one of the curves is determined by the wave parameter $D=4 R / \mathrm{ka}^{2}$ where $R$ is the propagation range, $k$ is the wave number, and $a$ is the correlation length.

An example of information to be gained from evaluation of the variances is given in Figure 2, which plots the two quantities

$$
\begin{gathered}
\gamma=\frac{u_{\mathrm{rms}}}{\phi_{\mathrm{rms}}} \\
\rho=\frac{\langle u \phi\rangle}{u_{\mathrm{rms}} \phi_{\mathrm{rms}}}
\end{gathered}
$$

where $u_{\mathrm{rms}}^{2}=\left\langle u^{2}\right\rangle$ and $\phi_{\mathrm{rms}}^{2}=\left\langle\phi^{2}\right\rangle$. Both $\gamma$ and $\rho$ are ratios that do not depend on the overall strength $\left\langle\mu^{2}\right\rangle$ of the inhomogeneities. For a medium that extends to depth $R$ with fluctuation statistics that are independent of depth, both $\gamma$ and $\rho$ depend on one parameter. Given a scale $a$ that characterizes the spectrum $W(\underline{K})$, that parameter is $D=4 R /\left(k a^{2}\right)$, which was defined as the wave parameter by Chernov [1960]. For each choice of medium spectrum there is a unique curve in $\gamma-p$ space traced out through different values of $D$. Some examples are given in Figure 2 along with some experimental results. It is seen that spectra of the power law type are a better fit to the variances than a Gaussian spectrum and that the values of $\rho$ cluster around 0.3 , while the values of $\gamma$ vary substantially. Note that higher seismic frequencies will tend to have lower $\gamma$ because $\phi_{\mathrm{rms}}$ increases with frequency faster than $u_{\text {rms. }}$. Of course, a medium whose spectrum is dependent in some way on depth will lead to $\rho, \gamma$ values that do not lie on these sample curves, but the general trend of smaller $\rho$ for power law spectra versus Gaussian spectra will apply.

Further information beyond the variances is available in coherence functions. For example, the phase coherence between two receivers at positions $\underline{x}_{1 T}$ and $\underline{x}_{2 T}$ can be expressed as

$$
\left\langle\phi\left(\underline{x}_{1 T}\right) \phi\left(\underline{x}_{2 T}\right)\right\rangle=\left\langle\phi\left(\underline{x}_{T}\right) \phi(0)\right\rangle
$$

where we have defined $\underline{x}_{T} \equiv \underline{x}_{2 T}-\underline{x}_{1 T}$ and we have used horizontal homogeneity of the spectrum. We define the transverse 
coherence functions (TCFs) corresponding to each of the above variances $\left\langle u\left(x_{T}\right) u(0)\right\rangle,\left\langle\phi\left(x_{T}\right) \phi(0)\right\rangle$, and $\left\langle u\left(x_{T}\right) \phi(0)\right\rangle$, respectively. Again, since horizontal homogeneity of the spectrum is assumed, these functions depend only on the difference between the two positions. The expression for the TCFs in terms of the medium spectrum are (Flatte and Moody, manuscript in preparation, 1987)

$$
\begin{gathered}
<u\left(\underline{x}_{T}\right) u(0)>=2 \pi k^{2} \int_{0}^{R} d z \int d^{2} \underline{K}_{T} W\left(0, K_{T}, z\right) \\
\sin ^{2}\left[K_{T}^{2} z / 2 k\right] \cos \left[\underline{K}_{T} \cdot \underline{x}_{T}\right] \\
<\phi\left(\underline{x}_{T}\right) \phi(0)>=2 \pi k^{2} \int_{0}^{R} d z \int d^{2} \underline{K}_{T} W\left(0, K_{T}, z\right) \\
\cos ^{2}\left[K_{T}{ }^{2} z / 2 k\right] \cos \left[\underline{K}_{T} \cdot \underline{x}_{T}\right] \\
<u\left(\underline{x}_{T}\right) \phi(0)>=2 \pi k^{2} \int_{0}^{R} d z \int d^{2} \underline{K}_{T} W\left(0, K_{T}, z\right) \\
\cos \left[K_{T}^{2} z / 2 k\right] \sin \left[K_{T}{ }^{2} z / 2 k\right] \cos \left[\underline{K}_{T} \cdot \underline{x}_{T}\right]
\end{gathered}
$$

Since $W$ is assumed not to depend on the direction of $K_{T}$ but only its magnitude (horizontal isotropy), the $K_{T}$ integral can be expressed in polar coordinates, and the angular integral may be done. The results are then a function only of the magnitude of the separation $\underline{x}_{T}$ and not its direction:

$$
\begin{gathered}
<u\left(x_{T}\right) u(0)>=4 \pi^{2} k^{2} \int_{0}^{R} d z \int_{0}^{\infty} K_{T} d K_{T} W\left(0, K_{T}, z\right) \\
\sin ^{2}\left[K_{T}^{2} z / 2 k\right] J_{0}\left(K_{T} x_{T}\right) \\
<\phi\left(x_{T}\right) \phi(0)>=4 \pi^{2} k^{2} \int_{0}^{R} d z \int_{0}^{\infty} K_{T} d K_{T} W\left(0, K_{T}, z\right) \\
\cos ^{2}\left[K_{T}^{2} z / 2 k\right] J_{0}\left(K_{T} x_{T}\right) \\
<u\left(x_{T}\right) \phi(0)>=4 \pi^{2} k^{2} \int_{0}^{R} d z \int_{0}^{\infty} K_{T} d K_{T} W\left(0, K_{T}, z\right) \\
\sin \left[K_{T}^{2} z / 2 k\right] \cos \left[K_{T}^{2} z / 2 k\right] J_{0}\left(K_{T} x_{T}\right)
\end{gathered}
$$

We find it convenient to work with normalized TCFs defined as follows:

$$
\begin{aligned}
\left.<\phi\left(x_{T}\right) \phi(0)\right\rangle_{N} & =\frac{\left\langle\phi\left(x_{T}\right) \phi(0)\right\rangle}{\left\langle\phi^{2}\right\rangle} \\
\left.<u\left(x_{T}\right) u(0)\right\rangle_{N} & =\frac{\left\langle u\left(x_{T}\right) u(0)\right\rangle}{\left\langle u^{2}\right\rangle} \\
\left.<u\left(x_{T}\right) \phi(0)\right\rangle_{N} & =\frac{\left\langle u\left(x_{T}\right) u(0)\right\rangle}{u_{\mathrm{rms}} \phi_{\mathrm{mss}}}
\end{aligned}
$$

These normalized functions, like the quantities $\gamma$ and $\rho$, do not depend on the overall strength $\left\langle\mu^{2}\right\rangle$ of the medium fluctuations. We note that the values of these coherences at $x_{T}=0$ are 1,1 , and $p$, respectively.

Note that our expressions for the variances and the TCFs are simpler than the usual Born approximation volume-integration expressions (S.M. Flatté and T. Moody, manuscript in preparation, 1987). Here we have a one-dimensional integral along $z$ and a one-dimensional integral over the spectrum. We see that
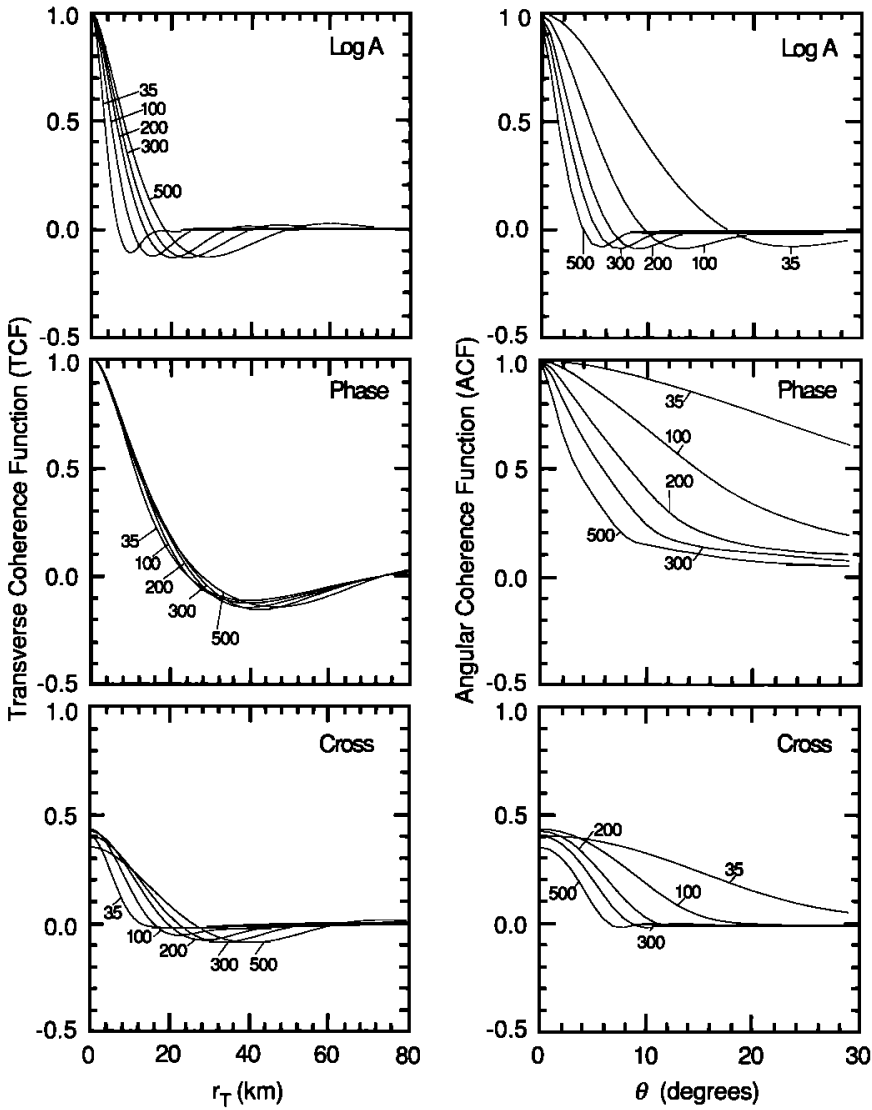

Fig. 3. The three transverse coherence functions (TCFs) and the three angular coherence functions (ACFs) for an extended medium with an exponential correlation function with scale $a=10 \mathrm{~km}$ and a seismic frequency of $1 \mathrm{~Hz}$. The different curves are results for media that extend from the surface to different depths given by the curve labels in kilometers: that is, varying from 35 to $500 \mathrm{~km}$.

the $\log$ amplitude fluctuation $u$ is weighted toward higher wave number $K_{T}$ than the phase fluctuation due to the "Fresnel filter" factors $\sin ^{2}\left[K_{T}^{2} z / 2 k\right]$ and $\cos ^{2}\left[K_{T}{ }^{2} z / 2 k\right]$. We also see that this weighting is more pronounced for smaller $z$; in other words, fluctuations of $u$ with wavelengths $\lambda_{K}>2 \pi(z / k)^{3 / 2}$ are suppressed. Note that there is more suppression near the surface; it is difficult for wave speed fluctuations to cause amplitude fluctuations without a sufficient drift space before the receiver.

It is helpful to show some examples of the normalized TCFs for an interesting medium spectrum. Figure 3 shows the three TCFs for a medium with an exponential correlation function with scale $a=10 \mathrm{~km}$. That is,

$$
W(K)=\frac{<\mu^{2}>a^{3}}{\pi^{2}} \frac{1}{\left(1+K^{2} a^{2}\right)^{2}}
$$

We see that the width of the phase coherence function is larger than the width of the medium correlation function, while the width of the $\log$ amplitude coherence function is smaller. This is due to the Fresnel-filter factor which favors high wave number and hence small scales in the log amplitude coherence.

\section{ANGULAR CORREL_ATION FUNCTIONS}

Consider teleseismic waves arriving from two different directions indicated by unit vectors $\boldsymbol{\theta}_{1}$ and $\boldsymbol{\theta}_{2}$; let the vectorial 


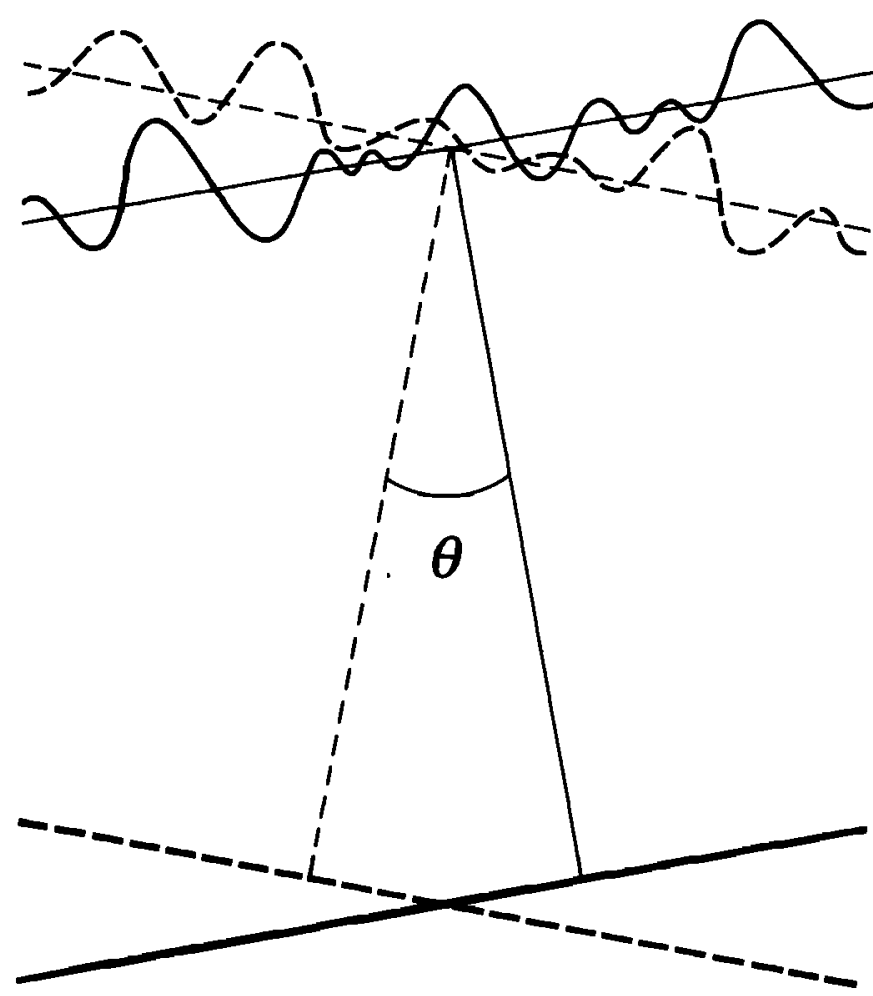

Fig. 4. Schematic of incident $\boldsymbol{P}$ waves from two different sources incident on a seismic array (see Figure 1).

difference in angle be $\underline{\theta}=\underline{\theta}_{2}-\underline{\theta}_{1}$. Finally, the magnitude of the angle between the two unit vectors is denoted by $\theta$. It is well known that the pattems of $u$ and $\phi$ on the Earth's surface will be different for waves from the two different directions, and this difference will be greater for larger angular differences: that is, larger $\theta$. (See Figure 4.) If we again make the assumption of horizontal homogeneity and isotropy of the medium fluctuation spectrum, then statistical correlation between the log amplitudes and phases of the waves from two different directions will be a function only of the magnitude of the angular difference between the two waves; that is, the magnitude of $\underline{\theta}$ and not its direction. We can then write (S.M. Flatte and T. Moody, manuscript in preparation, 1987):

$$
\begin{gathered}
<u(\theta) u(0)>=4 \pi^{2} k^{2} \int_{0}^{R} d z K_{T}^{\infty} d K_{T} W\left(0, K_{T}, z\right) \\
\sin ^{2}\left[K_{T}^{2} z / 2 k\right] J_{0}\left(K_{T} z \theta\right) \\
<\phi(\theta) \phi(0)>=4 \pi^{2} k^{2} \int_{0}^{R} d z \int_{T}^{\infty} K_{T} d K_{T} W\left(0, K_{T}, z\right) \\
\cos ^{2}\left[K_{T}^{2} z / 2 k\right] J_{0}\left(K_{T} z \theta\right) \\
<u(\theta) \phi(0)>=4 \pi^{2} k^{2} \int_{0}^{R} d z K_{T} d K_{T} W\left(0, K_{T}, z\right) \\
\sin \left[K_{T}^{2} z / 2 k\right] \cos \left[K_{T}^{2} z / 2 k\right] J_{0}\left(K_{T} z \theta\right)
\end{gathered}
$$

Thus these angular correlation functions (ACFs) are very similar to the TCFs except that the transverse separation $x_{T}$ has been replaced by $z \theta$; in other words, the transverse separation is a linear function of depth, given by the separation of two rays converging to the same point on the surface of the earth from the two different directions $\boldsymbol{\theta}_{1}$ and $\boldsymbol{\theta}_{2}$. (See Figure 4.)

The normalized ACFs have at least two important properties: First, they provide another set of observables from array data that give a different weighting to inhomogeneities at different depths than the TCFs and hence provide further important constraints on medium models. Second, the variable $\boldsymbol{\theta}$ depends on having at least two sources; since there are many more source (earthquake) locations than there are stations in a typical seismic array and since these earthquakes provide angular differences down to fractions of a degree, the ACFs provide a much finer resolution of the medium inhomogeneities than the TCFs. For example, the NORSAR subarrays are separated by distances of $10 \mathrm{~km}$ or more, so that the transverse coherence for separations $x_{T}$ smaller than about $10 \mathrm{~km}$ cannot be observed. However, observations with angular differences in incoming direction of about $1^{\circ}$ are not uncommon, providing a probe of scales of $1 \mathrm{~km}$ even at a depth of $60 \mathrm{~km}$.

Predictions for normalized ACFs for the medium spectrum of (22) (an exponential medium correlation function) are shown in Figure 3. Now the depth to which the medium extends becomes a more important factor in determining the curves. We see that the ACFs become narrower for media extending to larger depth if other parameters are kept the same.

\section{EXPERIMENTAL RESULTS}

Previous results for the variances have been given by $A k i$ [1973], Capon [1974], Berteussen et al., [1975a, b], Powell and Meltzer [1984], and others. In many cases they presented their results in terms of a uniform, isotropic random layer with a Gaussian spectrum. We have inverted the relevant equations where necessary to revive their variance data with no imposition of a medium model. In cases where event-by-event results were given, we have averaged all the events presented. The results are given in Table 1 . We see general agreement between the various determinations when expressed in this form, except for a few cases where a very small number of events were used. Variance results are graphed in Figure 2, where it is seen that individual events have a reasonably large scatter; averages are consistent with a power law type of medium rather than a Gaussian spectrum.

Previous results for TCFs were restricted to the log amplitude and phase without the cross-coherence TCF. Aki [1973] and Capon [1974] have presented approximate TCFs from LASA data showing decorrelation scales of the order of $10 \mathrm{~km}$.

Previous results for ACFs consist only of qualitative comments. Berteussen [1975] remarks that events are well correlated in amplitude fluctuations if they are within a slowness difference of $0.5 \mathrm{~s} / \mathrm{deg}$, which implies that their incoming direc-

TABLE 1. Experimental Values for Variances

\begin{tabular}{lccc}
\hline \multicolumn{1}{c}{ Reference } & $\tau_{\text {rms }} s$ & $u_{\text {rms }}$ & $\rho$ \\
\hline Aki [1973] & 0.13 & 0.32 & 0.35 \\
Capon [1974] & 0.10 & 0.40 & - \\
Berteussen et al. [1975a, b] & $0.006-0.11$ & $0.2-0.4$ & - \\
This work & $0.135 \pm 0.004$ & $0.410 \pm 0.006$ & $0.26 \pm 0.03$ \\
\hline
\end{tabular}




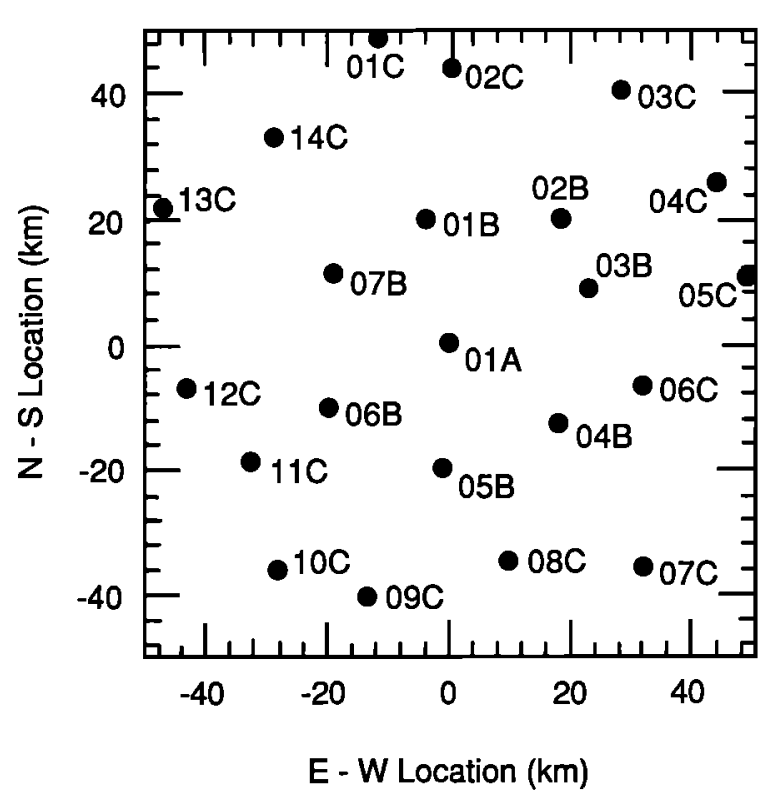

Fig. 5. Subarray spatial distribution at NORSAR. Subarray $01 \mathrm{~A}$ is at latitude $60.82 \mathrm{~N}$ and longitude $10.83 \mathrm{E}$.

tions are within about $2^{\circ}$ and gives an example showing that two events are substantially decorrelated if they are $7^{\circ}$ apart in azimuth, which implies that their incoming directions are $5^{\circ}$ apart.

There are many other publications that include attempts to fit a deterministic structure to the data from one or many events; we will not try to discuss their data, as it would have been presented in such a different way that the observations relevant to our attempts to observe small scale structure would not have been given. We discuss the relationship of our results to some of the deterministic results of others in section 6.

We now discuss our presentation of the NORSAR subarray data given in two NORSAR reports by Berteussen [1974] and Berteussen and Husebye [1974]. The data have been filtered in two important ways. First, a frequency filter has restricted the seismic frequency to $1-3 \mathrm{~Hz}$, so the nominal center frequency is $2 \mathrm{~Hz}$. Second, the elements within the subarrays have been added coherently; each subarray has about six elements spread over a circle of radius $-3.5 \mathrm{~km}$. The spatial distribution of subarrays is shown in Figure 5.

The travel time anomalies given by Berteussen [1974] are given for 104 beams; each beam is an average over a number of events whose source locations are close such that their incoming directions at the NORSAR array are within a few tenths of a degree. The travel time anomalies consist of arrival time residues with respect to a "best plane wave." We have removed the mean arrival time for each beam in our calculations. Figure 6 shows the arrival angle distribution of these beams.

The subarray intensity variations are given by Berteussen and Husebye [1974] for 185 beams. We have normalized these data such that for each beam the mean log amplitude is zero.

With this brief description of the gathering of the data (more details are given in the original reports) we now describe our method of estimating variances, TCFs and ACFs. More details, including our method of estimating the associated statistical errors, are given in the appendix.

If we had subarrays that were far apart and beams that were far apart in angle, then the information from each subarray and from each beam would be statistically independent. The subarrays are far enough apart in location that they are reasonably independent for the purposes of error calculations. However, the beams are often close together, and hence the assumption of independent beams is not valid. If the beams are not independent, then the calculation of any quantity should use an appropriate weight for each beam. Let $b$ be an index over the $N$ beams; then we define $w_{b}$ as the weight of beam $b$. The appendix describes our form for the weight. In order to calculate reasonable errors, we must establish the effective number of independent beams; this is also done in the appendix. We note that the number of independent beams is different for the different TCFs; we find 42.1, 11.5, and 32.4 for the effective number of independent beams in determining the TCFs of $\log$ amplitude, phase, and their cross-TCF, respectively. The ACFs have similar numbers, although the particular number varies with the angle.

To describe our formulas for the determination of experimental quantities, consider first the variances. We have

$$
\begin{aligned}
\left.<u^{2}\right\rangle & =\frac{1}{N_{w}} \sum_{b=1}^{N} w_{b} \frac{1}{M} \sum_{s=1}^{M}\left(u_{b s}\right)^{2} \\
\left.<\phi^{2}\right\rangle & =\frac{1}{N_{w}} \sum_{b=1}^{N} w_{b} \frac{1}{M} \sum_{s=1}^{M}\left(\phi_{b s}\right)^{2} \\
<u \phi\rangle & =\frac{1}{N_{w}} \sum_{b=1}^{N} w_{b} \frac{1}{M} \sum_{s=1}^{M}\left(u_{b s} \phi_{b s}\right)
\end{aligned}
$$

where $b$ is an index over the $N$ beams, $N_{w}=\sum w_{b}$, and $s$ is an index over the $M$ subarrays. The only difficulty in the above calculations is that the sets of beams for $u$ and $\phi$ are not identical. In order to calculate the cross coherence, we have searched through the two sets of beams and found corresponding pairs that are within $1^{\circ}$ of each other and have considered them the same beam. The calculation of the errors is somewhat involved

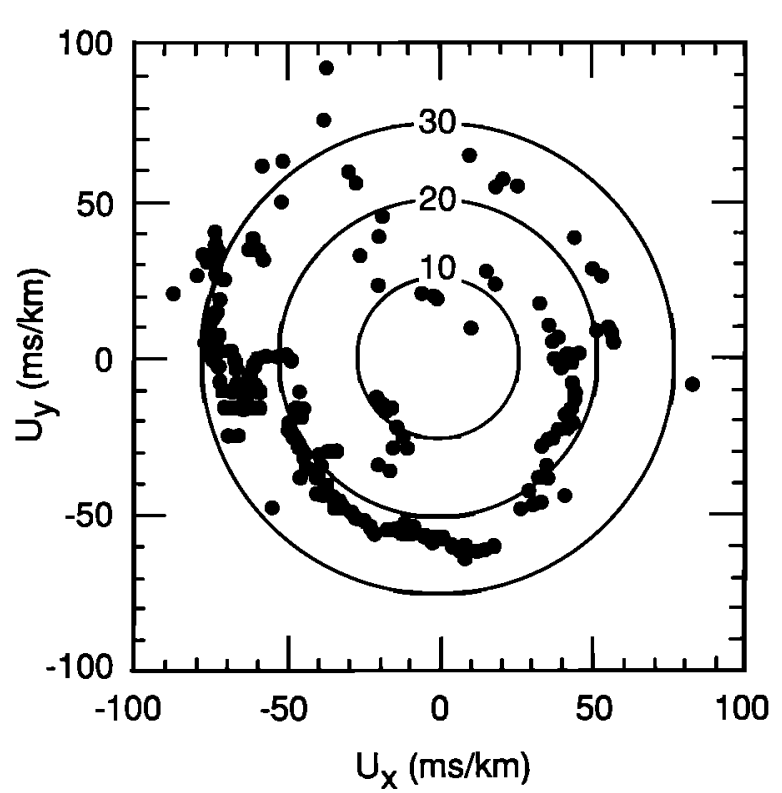

Fig. 6. Arrival angle distribution of the various beams defined at NORSAR. Each beam represents data from a cluster of earthquakes that are close enough that their seismic waves arrive at NORSAR with angles that are within a few tenths of a degree. The circles are labelled with the angle from the zenith in degrees. The axes are labelled with the components of slowness. 

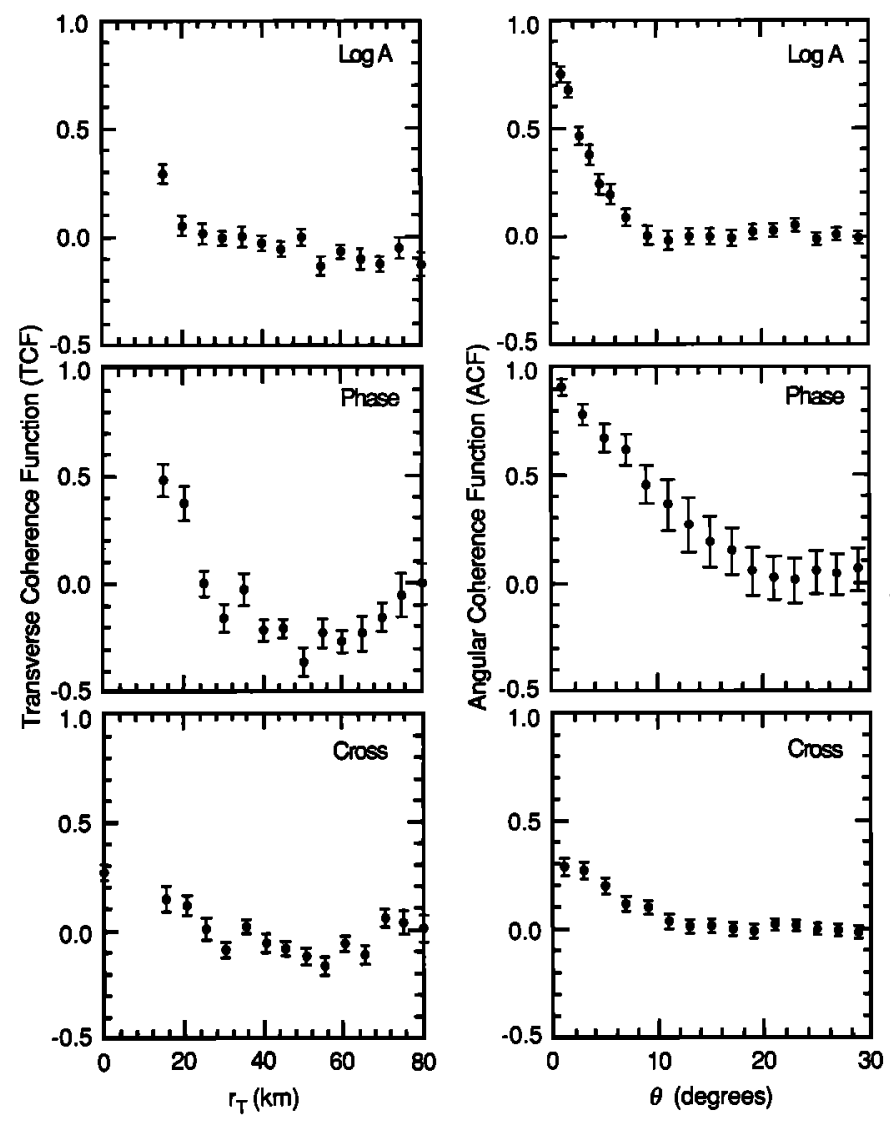

Fig. 7. Observed normalized TCFs and ACFs at NORSAR. Note that the regions of rapid variation in the TCFs are not covered well by the data, because the different subarrays have a minimum separation of about $15 \mathrm{~km}$; the regions of rapid variation in the ACFs are well covered by the data, because the minimum separation in angle between the different beams is in the range of a few tenths of a degree.

and is given in the appendix. The results of the calculations of (26)-(28) are given in Table 1.

The TCFs are calculated with formulas analogous to (26)(28). In order to plot a point at a transverse separation $x_{T}$, we include in the sum all pairs of elements with their separation $x_{T}$ within a given bin. The results of this procedure are shown in Figure 7. We see that the smallest separation available is about $15 \mathrm{~km}$, and the coherence is already below 0.5 at that separation.

Finally, we calculate the ACFs by the same method, with the order of summation reversed and with selection of pairs of beams whose angular difference is within a specified bin. The ACFs are also shown in Figure 7. We see that the experimental points in the ACFs have as much statistical precision and more resolution within the variable range for which there is significant variation in the coherence than the TCFs. The higher resolution of the ACFs compared with the TCFs follows from the closeness in arrival angle of the beams as compared to the spatial separation of the subarrays.

Certain dramatic features of the ACFs provide constraints on earth structure models. The phase coherence function has a smooth almost linear drop to a small coherence at an angle of about $15^{\circ}$. The $\log$ amplitude coherence has a distinctively different shape than the phase coherence; it drops sharply to 0.5 at an angle of about $2^{\circ}$ but retains significant correlation out to angles of order $10^{\circ}$. We shall see in the next section the implications of these features for Earth models.
It is to be emphasized at this point that the nature of the NORSAR array restricts the scales of Earth structure that can be observed with NORSAR data. The approximate diameter of the array is $110 \mathrm{~km}$. Because means and "best plane waves" have been subtracted from the data across the array, we cannot observe Earth structure with scales larger than about $110 \mathrm{~km}$. Because the data consist of coherent addition of stations over subarrays that are about $7 \mathrm{~km}$ in diameter, we cannot observe structure with wavelengths smaller than about $5 \mathrm{~km}$. Furthermore, our typical seismic wavelength at $2 \mathrm{~Hz}$ of $4 \mathrm{~km}$ also implies that we cannot observe structure wavelengths less than $-4 \mathrm{~km}$. These facts have to be taken into account in the theoretical models with which we compare the experimental data.

It is true that the separations of stations within subarrays are in the 1- to $7-\mathrm{km}$ range. We have obtained station data for 13 nuclear explosion events of high signal-to-noise ratio at NORSAR. We have found that the station-by-station time anomalies are less than $0.1 \mathrm{~s}$ and are difficult to measure reliably because of the differing signal temporal structures. The signal intensities are more reliable; we have taken a 5-s time window at the start of the signal and have calculated the log amplitude TCF in the same manner that we did for the subarray data. The results will be shown later when we compare models to the data.

It is of interest to note that the results of this and the previous section, that is, the TCFs and the ACFs, can be used to understand the errors and possible biases of sparse arrays in measuring the magnitude of an earthquake or nuclear explosion. The analysis technique of McLaughlin et al. [1988], along with our experimentally determined coherence functions, could be applied to this problem.

\section{SPECTRAL MODELS OF EARTH STRUCTURE UNDER NORSAR}

We picture the crust, lithosphere, and asthenosphere below the Earth's surface as having random variations in seismic wave speed that are described by a spectrum that may depend on depth and may be anisotropic. We assume that the spectrum does not depend to first order on small changes in geographical position within the region under the NORSAR seismic array. The most desirable situation would be one in which we had enough experimental information to invert the data for all the characteristics of the spectrum describing these variations. We do not delude ourselves into thinking that our present data set is adequate for the task. However, we can impose some constraints on these type of models by analysis of the data, and we can find an Earth model which is at least consistent with the data and with what we know of the Earth's structure from other information.

We need to modify our theoretical expressions for the fact that the NORSAR array has a finite size and for the fact that the NORSAR data are averaged over subarrays and is at finite nonzero wavelength. The finite size is modeled by removing from the spectrum all wavelengths greater than $110 \mathrm{~km}$. There is a considerable uncertainty in exactly where this upper wavelength cutoff should be because it depends on exactly how the "best plane waves" were removed and how averages of log amplitude were made; the main comment to make is that this cutoff should be arranged to fit the coherence functions at large separations; as a result the observed large-separation coherences are not a strong constraint on Earth models. This cutoff does not introduce artifacts into the coherence functions at small transverse separations or at small angular separations. 
The subarray averaging might be modeled by assuming that the data are averaged uniformly over the area of a circle of radius $r_{0}=3.5 \mathrm{~km}$. This convolution corresponds in the spectral domain with multiplying our spectra by the following function of $K_{T}$ :

$$
C\left(K_{T}\right) \equiv\left[\frac{2 J_{1}\left(K_{T} r_{0}\right)}{K_{T} r_{0}}\right]^{2}
$$

This function has its first zero at $K_{T} r_{o}=3.8$, or a mediumwavelength scale of $5.5 \mathrm{~km}$. In our theoretical models we use a small-wavelength cutoff of $5.5 \mathrm{~km}$. Again, this cutoff does not introduce artifacts into the coherence functions, but it does affect their exact shape at very small separations (of order $5 \mathrm{~km}$ or $1^{\circ}$ ); future investigation of individual station waveforms will allow a more rigorous treatment of these cutoffs.

The first model we may try is one with an exponential correlation function with a scale length of $10 \mathrm{~km}$, following those authors who have looked only at TCFs [Aki, 1973; Capon, 1974; Capon and Berteussen, 1974; Berteussen et al., 1975a, b; Powell and Meltzer, 1984]. (See Figure 8.) We see that the TCF data and the ACF of phase are not badly fit with medium variations extending down to about $200 \mathrm{~km}$, but the ACF of $\log$ amplitude and the cross ACF have significant disagreements between model and data. The disagreements have to do with the shapes of the ACFs in the regions of their rapid variations: at angles less than $10^{\circ}$. We should point out that disagreements at
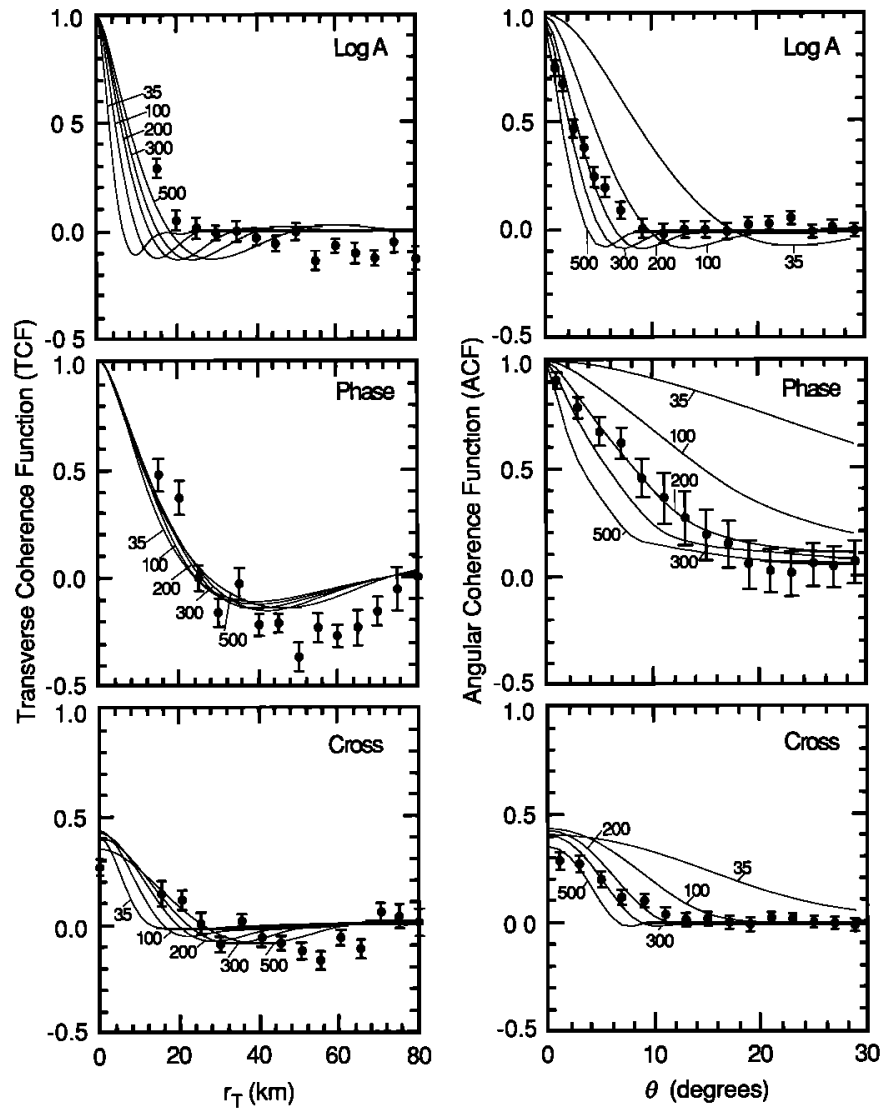

Fig. 8. Comparison between the data and calculations based on uniform random extended media with exponential correlation functions having a scale length of $10 \mathrm{~km}$. The media extend from the surface to depths of 35 to $500 \mathrm{~km}$. Note that the best medium extent for the ACF of phase is about $200 \mathrm{~km}$, but that model does not give a good fit to the shapes of the ACF of log amplitude and the cross ACF.
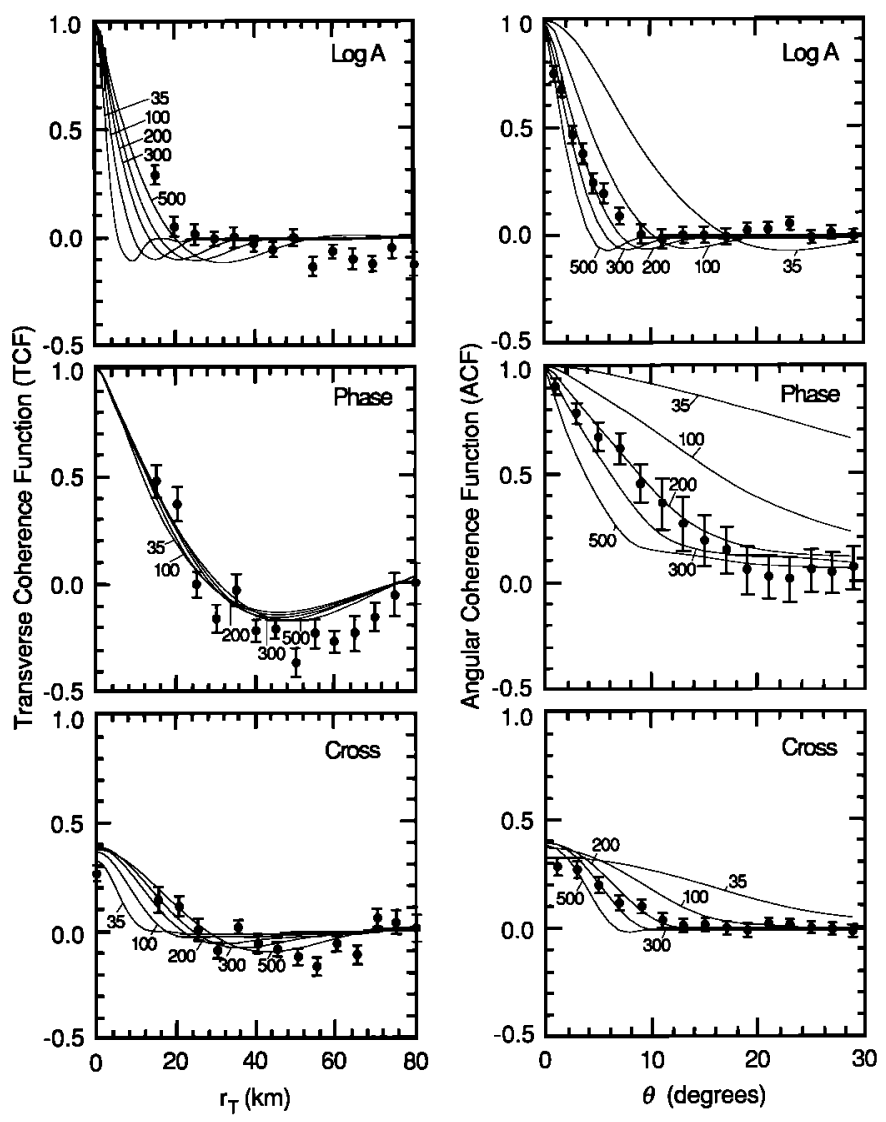

Fig. 9. Comparison between the data and calculations based on uniform random extended media with power-law spectra having index $p=3$ (self-similar). The media extend from the surface to depths of 35 to 500 km. As in Fig. 8, the best medium extent for the ACF of phase is about $200 \mathrm{~km}$. This self-similar model gives a better fit than the exponential correlation model, but the self-similar model still does not fit the shapes of the ACF of log amplitude and the cross ACF.

large angles are probably less important because the shapes of the functions there are affected by the finite aperture of the NORSAR array. It is of interest even in this rather unsatisfactory model that structure is necessary down to several hundred kilometers to give a fit with reasonable average scale lengths.

The data in Figure 2 indicate that a power law spectrum is also appropriate for comparison to the data (in this case, $\gamma$ and p). We will therefore restrict further models to a particular type of spectrum: a power law expressed in the form

$$
W(\underline{K})=A K^{-p}
$$

There is a wide variety of models with power law spectra because the power law index $p$ may change with depth, as may the strength $A$. We have tried many examples of uniform media down to a cutoff depth, but none of the fits were judged to be acceptable. For example, Frankel and Clayton [1986] suggested the use of a uniform layer with a power law medium with $p=3$, which they refer to as a self-similar medium. Figure 9 shows the predictions of such a medium; we see that the fit is better than that of the exponential medium, but some disagreements remain in the ACF of $\log$ amplitude and the cross ACF at angles less than $10^{\circ}$.

We have found one combination of power law media that we feel does fit the data reasonably well. We have not yet completed a full inversion to obtain a quantitative idea of the param- 


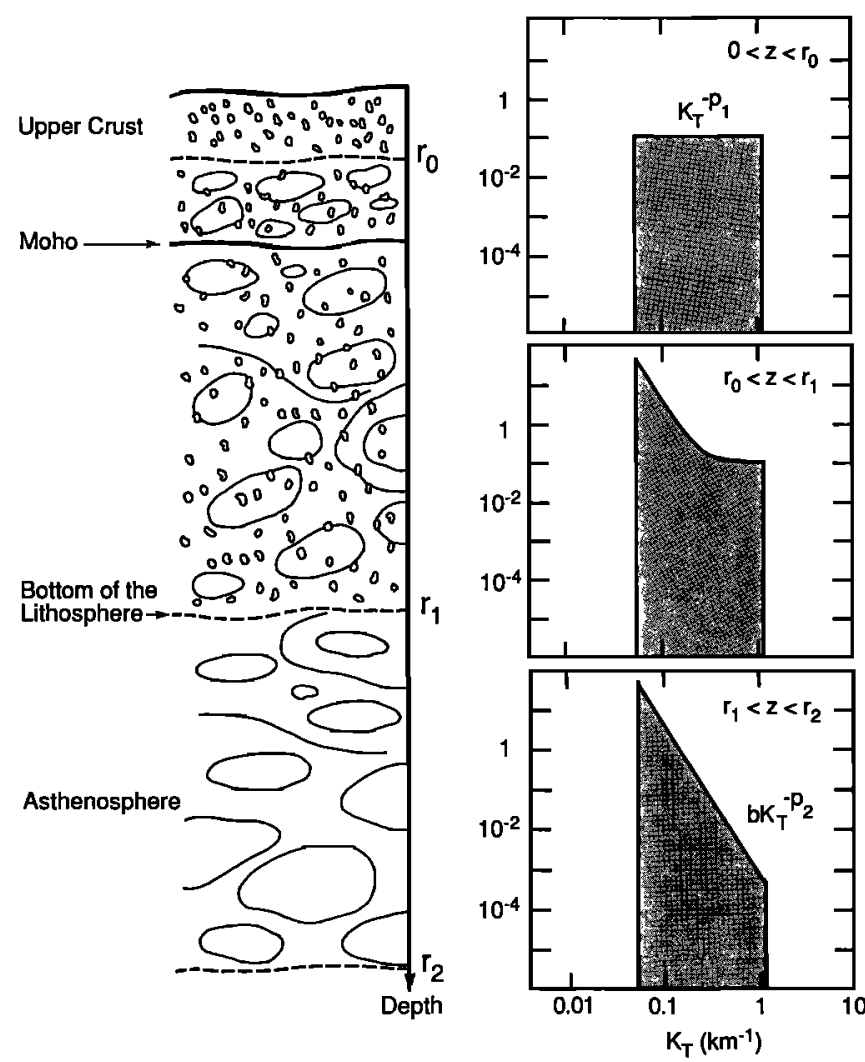

Fig. 10. Schematic representation of our best model of random variations under the NORSAR array. The model spectra have sharp cutoffs at low and high wave number to simulate the effects of the finite array aperture, the finite size of the subarrays, and the finite seismic wavelength.

eter uncertainties in this model. Our best model consists of two overlapping layers (Figure 10). The first layer from the surface down to 200-km depth has a power law index $p=0$ : in other words, a band-limited white spectrum. The second layer lies between depths of 15 and $250 \mathrm{~km}$ and has a power law index $p=4$ and a strength such that the $p=0$ and $p=4$ spectra cross at $K=0.31 \mathrm{~km}^{-1}$. Note that $p=4$ corresponds to the high wave number behavior of an exponential correlation function.

Comparison of the data and our best model is shown in Figure 11. One important feature of the data that constrains the model is the rapid decrease in the log amplitude ACF compared with the phase ACF. Most single-layer models have a similar shape for the two ACFs; in our best model, the log amplitude ACF is controlled by the shallow, flat spectrum layer, and the phase ACF is controlled by the deep, steep spectrum model.

Figure 12 shows the predictions of several variations on our best model: the shallow layer alone, the deep layer alone, the result of extending the shallow layer to $250 \mathrm{~km}$ instead of only $200 \mathrm{~km}$, and the result of extending the deep layer up to the surface instead of only to $15-\mathrm{km}$ depth. The fits are clearly inferior to the best model, although the differences of the latter two examples from our best fit model are not terribly striking.

The $\mathrm{ms}$ variations in phase and $\log$ amplitude are directly proportional to the $\mathrm{rms}$ variation in $P$ wave velocity in our model. However, they are also proportional to the square root of the medium correlation length in the vertical, which we have not measured. If we assume that the medium spectrum is isotropic, then taking the observed ms value of $u$ as 0.41 , we deduce an
Ims variation in $P$ wave velocity of $0.9 \%$ in the upper layer and $0.5 \%$ in the lower layer. If we use the observed ms value of $\phi$, which is 1.70 , we find values of 2.2 and $1.3 \%$. We regard the difference between the values obtained from log amplitude variance and from arrival time variance as not very significant because the determination of overall variance, as opposed to spectral level, is notoriously difficult [Flatte et al., 1979]. The difference does imply that our result for $\operatorname{mms} \boldsymbol{P}$ wave velocity fluctuation must be stated with large uncertainty; that is, it is between 1 and 4\%, if an isotropic spectrum is assumed. However, it does not seem likely that isotropy is appropriate. If we define the anisotropy ratio as the horizontal correlation length over the vertical correlation length, then our result for the velocity fluctuation is proportional to the square root of that ratio. The fact that the upper and lower layers have Ims variations within a factor of 2 of each other indicates that neither are second-order effects; both layers are significant in determining the wave fluctuations observed in the seismic array.

The predictions for the phase and cross TCFs fit the data reasonably well, but the $\log$ amplitude TCF prediction appears, mainly because of one data point, to decrease more rapidly than the data. After observing this discrepancy we used 13 nuclear explosion events at NORSAR to determine a more accurate experimental log amplitude TCF. (See section 4.) This TCF
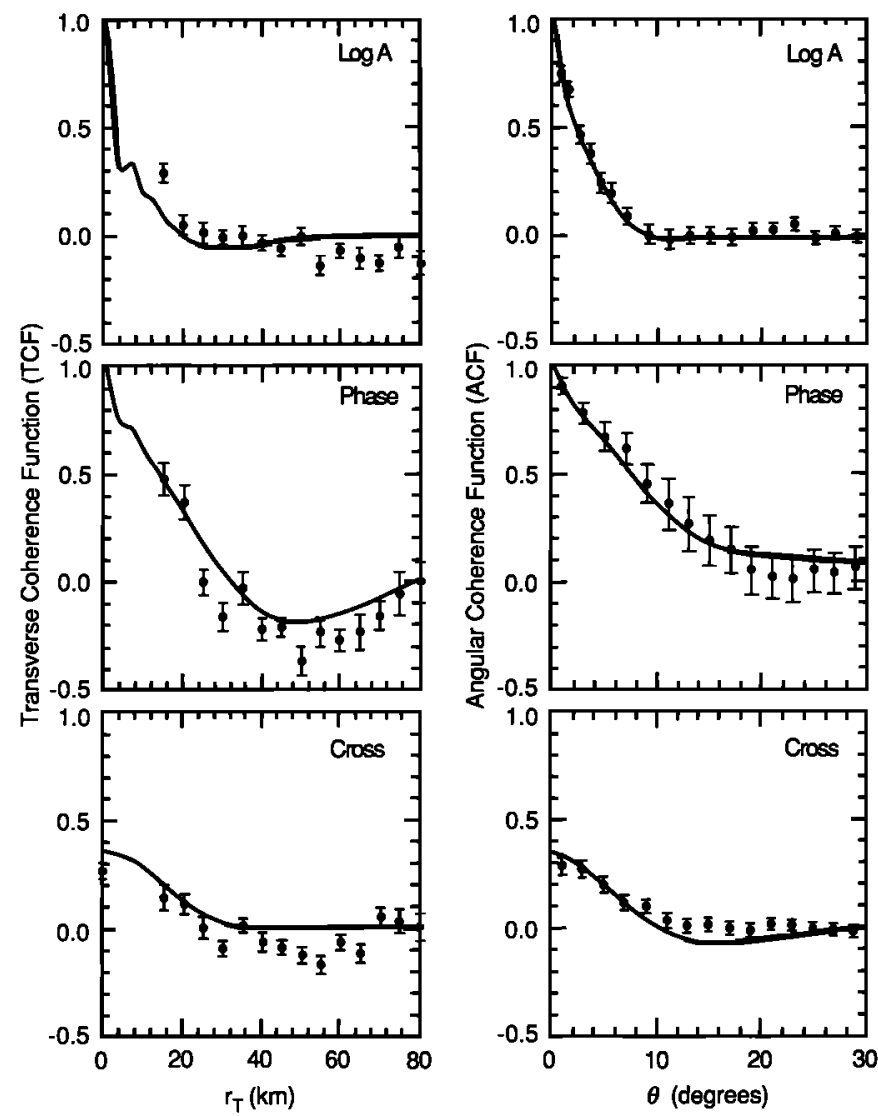

Fig. 11. Comparison between the data and the predictions of our best model, consisting of two overlapping layers. Each layer has a power law spectrum characterized by spectral index $p$. The characteristics of the two layers are $(0<z<200 \mathrm{~km} ; p=0)$ and $(15<z<250 \mathrm{~km}$; $p=4$ ) with nomalization such that in the overlapping depth region they have equal spectral levels at wavenumber $0.31 \mathrm{~km}^{-1}$. The fit is quite reasonable, particularly in the regions of rapid variation (less than $10^{\circ}$ in the ACFs). 

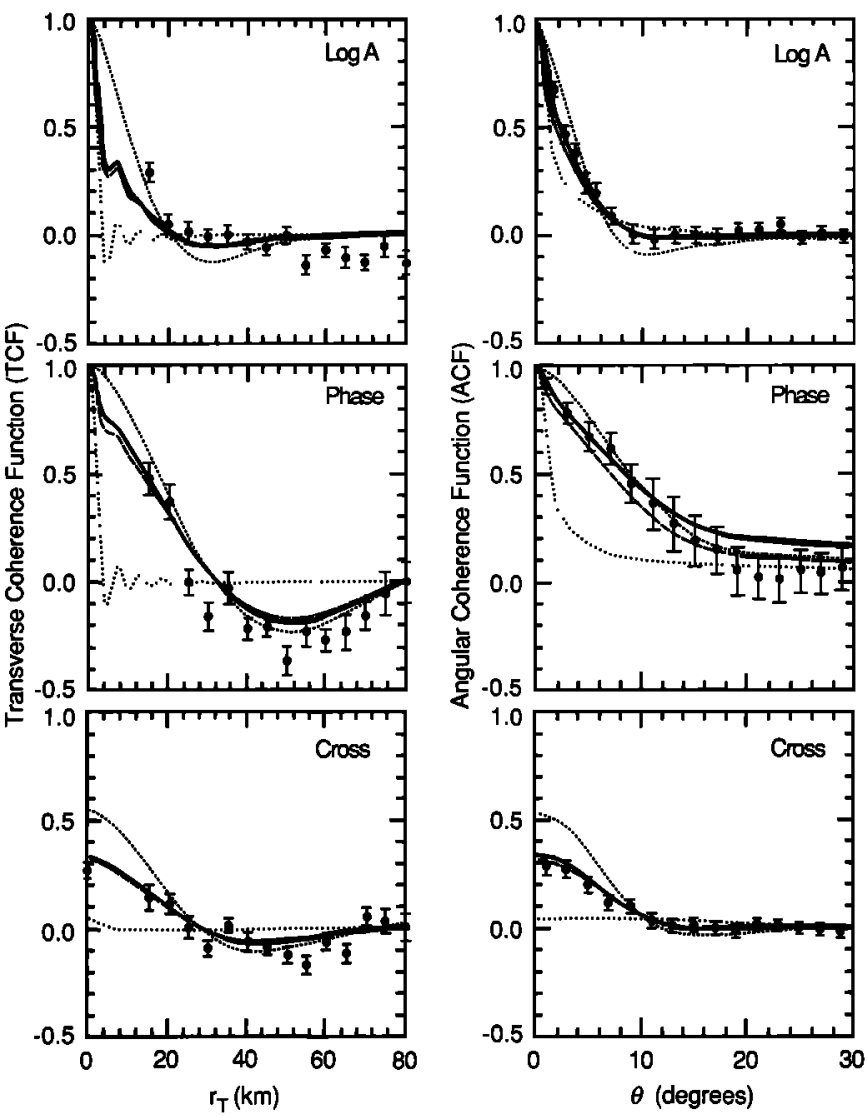

Fig. 12. Comparison between the data and the predictions for variations taken from our best model. Short dashes: the deep layer only; dotted: the shallow layer only; long dashes: our best model modified by extending the shallow layer to a depth of $250 \mathrm{~km}$; solid: our best model modified by extending the deep layer up to the surface.

involves single seismometer stations rather than subarray averages and hence involves separations down to $3 \mathrm{~km}$. We were gratified to observe the agreement between our best model prediction and this data set obtained after we had developed our best model mostly from the ACFs. (See Figure 13.)

\section{GEOPHYSICAL INTERPRETATION OFTHE OBSERVED MEDIUM VARIATIONS}

The Earth is characterized by vigorous geological activity driven by convection in the mantle. It is believed that most of the temperature increase with depth in the Earth occurs within thin boundary layers at the top and bottom of the mantle. But this is a statement about averages, and numerical simulations of mantle convection have indicated that strong variations may occur in these boundary layers [Boss and Sacks, 1985; Olson et al., 1987]. These variations may involve temperature differences of up to $1000 \mathrm{~K}$, with spatial scales perhaps comparable to the postulated boundary layer thicknesses of order $100 \mathrm{~km}$. The variations may also involve compositional differences or, in the upper mantle, variations due to partial melting. Although there is a wide variety of possibilities for the formation of Earth inhomogeneities, we think it helpful to note that our observations can be interpreted as small-scale variations driven by dynamic mantle convection, or as fossilized compositional differences, perhaps induced by convective processes in earlier eras.

The change from the shallow layer with its abundant small- scale structure to the deep layer with predominantly larger-scale variations could be indicative of some change in rock strength. One way to obtain small-scale structure is from a statistical distribution of cracks, but it seems unlikely that cracks would extend to $100-\mathrm{km}$ depths. Another way would be to have a random distribution of relatively homogeneous layers with discontinuities in $P$ wave speed from one layer to the next: layers whose thickness, horizontal extent, and wave speed discontinuity across interfaces are statistical in nature. Our results would imply that these random layers do not persist below about 200-km depth.

The ms variation in seismic wave speed that is implied by our spectral model is a few percent over the wavelength band $5.5-110 \mathrm{~km}$, which is obtained from the variance of phase or $\log$ amplitude. This small variation, which has been obtained by previous workers as well, is not inconsistent with known properties of materials combined with the above geophysical parameters.

We may note some relationships of our results to previous studies of deterministic structure in the same depth regime. All of these studies deduced structure whose lateral variations are at scales larger than $100 \mathrm{~km}$. Our data discriminate against structure with lateral wavelengths greater than $110 \mathrm{~km}$. Therefore our results are complementary to the following deterministic studies, whose results should in some cases be geologically related to ours.

Haddon and Husebye [1978] constructed a model with a single, deep dipping layer (thin lens model), calculated its predictions by the parabolic wave equation, and compared them with the spatial structure of arrival time and amplitude across the NORSAR array. They concluded that a layer at a depth of $150-200 \mathrm{~km}$ explains a good fraction of the variances. Their

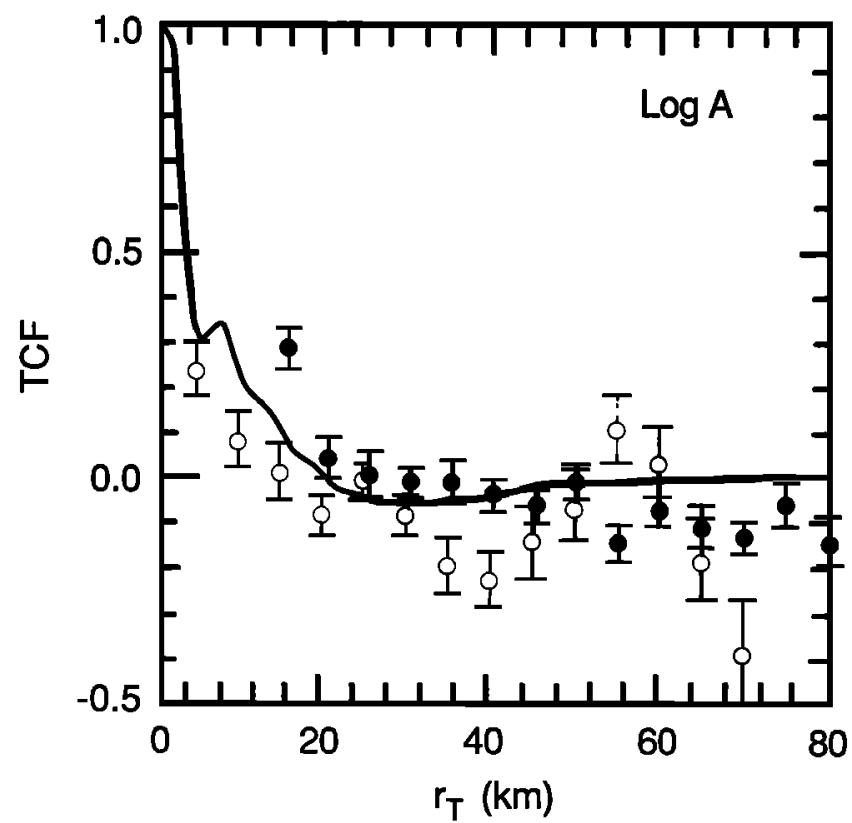

Fig. 13. The TCF of $\log$ amplitude for 13 nuclear explosion events (eight from the Soviet Union, five from the United States) detected at NORSAR (open circles). The data were calculated from individualstation waveforms; note that the individual stations have separations that range down to about $3 \mathrm{~km}$. We see that when the resolution is available, the coherence function drops to less than 0.5 in a few kilometers. For comparison, the solid circles are the same points shown in Figure 7, which represent subarray-averaged data. 
amplitude data had been smoothed such that only the large-scale systematic variation was kept. Therefore their results are complementary to ours.

Sacks et al. [1979] deduce a discontinuity at $250 \mathrm{~km}$ from observations of long-period precursons to direct $S$ at NORSAR. They suggest the precursors result from $S$ to $P$ conversion at the discontinuity, which they assume to be the lithosphereasthenosphere boundary. Our results also show that $250 \mathrm{~km}$ is an interesting depth. We would rather favor the interpretation that $250 \mathrm{~km}$ is the bottom of a thermal boundary layer in the mantle, with the bottom of the lithosphere being at $200 \mathrm{~km}$.

Given and Helmberger [1980] deduce laterally independent structure of $P$ wave velocity as a function of depth for northwest Eurasia, using short-period and long-period body waves from nuclear explosions. They see a low-velocity zone in the regime of $150-200 \mathrm{~km}$, which is likely to be geologically related to our observations of changes at 200 and $250 \mathrm{~km}$.

Husebye et al. [1986] reported a seismic tomographic survey of the lithosphere-asthenosphere beneath southern Scandinavia using travel time residuals. They placed the boundary between the lithosphere and asthenosphere between 100 and $200 \mathrm{~km}$, with lateral variations of scale greater than $100 \mathrm{~km}$.

Thus other studies of the larger-scale lateral variations under NORSAR have indicated structure at depths of $150-250 \mathrm{~km}$, which should be combined with our observations of smallerscale lateral variations down to the same depth range in order to create a geologically consistent picture.

Present ability to model the detailed dynamics of mantle convection is so crude that as yet no meaningful comparison between the strength and spectrum of variations that we observe and the expected product of mantle convection is possible. However, one can hope that future understanding of mantle convection, perhaps involving numerical simulation, will be influenced by measurements such as ours of the strength and spectrum of seismic wave speed variations in the thermal boundary layers of the mantle. More generally, improved geological understanding of the crust and lithosphere may come from requiring agreement with the small-scale variations that we observe in our shallow layer.

\section{APPENDIX}

TCFs and Their Statistical Errors

We begin the treatment of error estimates for the TCFs by considering the expression for the TCF of log amplitude obtained from the data of one beam:

$$
c_{b}\left(x_{T}\right)=\frac{1}{M} \sum_{p=1}^{M} u_{b s} u_{b k}
$$

where $p$ is an index over the $M$ pairs of subarrays whose separation is within the desired bin of $x_{T}$. (The index of the first member of the pair is $s$; the index of the second member is $t$.) The approximate variance of $c_{b}$ is obtained as follows: First, the expectation value of $c_{b}$ is needed,

$$
<c_{b}>=\rho_{l}\left(x_{T}\right)
$$

where $p_{t}$ is the unnormalized TCF at $x_{T}$. Next, the expectation value of the square is needed,

$$
<c_{b}^{2}>=\frac{1}{M^{2}} \sum_{p q} \sum<u_{b s} u_{b t} u_{b m} u_{b n}>
$$

Our assumption of Gaussian variables allows us to evaluate the above fourth moment in terms of all possible permutation of products of second moments [Flatte et al., 1979]. The result is then

$$
<c_{b}^{2}>=\rho_{l}^{2}+\frac{1}{M}<u^{2}>^{2}
$$

where we have ignored many of the off-diagonal coherences as small compared with the variance of $u$. Thus the variance of $c_{b}$ is

$$
\sigma_{b}^{2}=\frac{1}{M}<u^{2}>^{2}
$$

It is important to note that because we have many beams, we can observe $\sigma_{b}$ at each $x_{T}$. We simply plot the distribution of $c_{b}$ from the many beams and calculate the variance of the distribution. The question is, what is the uncertainty in $c_{b}$ after combining all the information from the many beams? We will couch this in the form of the question of determining $N_{e f f}$, the effective number of independent beams.

Since we have data from $N$ beams, we should combine them in an appropriate manner; that is, with weights:

$$
c\left(x_{T}\right) \equiv\left\langle u\left(x_{T}\right) u(0)\right\rangle=\frac{1}{N_{w}} \sum_{b=1}^{N} w_{b} c_{b}\left(x_{T}\right)
$$

where $b$ is an index over the $N$ beams, $w_{b}$ is the weight assigned to each beam, and

$$
N_{w}=\sum_{b=1}^{N} w_{b}
$$

If the $N$ beams were independent, then the optimal combination would require $w_{b}=1$, and it is simple to show that the variance of $c$ is given by

$$
\sigma^{2}=\frac{1}{N M}<u^{2}>^{2}
$$

We define the number of effective beams as

$$
N_{\text {off }} \equiv \frac{\sigma_{b}^{2}}{\sigma^{2}}
$$

That is, in order to calculate the variance of our measurement of the TCF at a particular $x_{T}$, we observe the distribution of that TCF from our many beams, determine its variance from that distribution, and then divide by $N_{\mathrm{ofr}}$. For the case of independent beams, from (A5) and (A8) we see that $N_{\text {eff }}$ is equal to the total number of beams $N$.

However, many of the beams in this data set are so close to each other that the information in each is not independent. Therefore a more accurate measure of the TCF is obtained by weighting each beam by a number smaller than unity if there are nearby similar beams. We have chosen weights for each beam in the following way:

$$
w_{b}=\frac{1}{1+\sum_{d \rightarrow b}^{N} p_{a}\left(\theta_{b d}\right)}
$$

where $p_{a}\left(\theta_{b d}\right)$ is the ACF between beams and $\theta_{b d}$ is the angle between beam $b$ and beam $d$. This weighting has the appropriate limits; first, it is equal to unity if all beams are far from each other and therefore independent; second, if there are $n$ beams on top of each other, each beam has a weight of $1 / n$.

Now we must estimate the statistical error on this coherence function. We emphasize that our treatment of errors makes significant approximations; it is very difficult to be more accu- 
rate in the evaluation of errors without going through an extensive program of numerical simulation. We assume that all the beams are in a narrow cone, so that the expectation value of the TCF for each beam is the same, and we assume that the statistics of the log amplitude or arrival time are close enough to Gaussian to estimate the error reasonably. Let us use a shorthand notation in which $x_{T}$ is suppressed:

$$
c=\frac{1}{N_{w}} \sum_{b=1}^{N} w_{b} c_{b}
$$

and we need to find an appropriate expression for $\left\langle c^{2}\right\rangle$.

$$
\left\langle c^{2}\right\rangle=\frac{1}{N_{w}^{2}} \sum_{b=1 d=1}^{N} \sum_{d=1}^{N} w_{b} w_{d}\left\langle c_{b} c_{d}\right\rangle
$$

But each $c_{b}$ is the sum of products of two $\log$ amplitudes. We may indicate this by

$$
\left\langle c_{b} c_{d}\right\rangle=\frac{1}{M^{2}} \sum_{i=1}^{M} \sum_{j=1}^{M}\left\langle u\left(x_{i}, \theta_{b}\right) u\left(y_{i}, \theta_{b}\right) u\left(x_{j}, \theta_{d}\right) u\left(y_{j}, \theta_{d}\right)\right\rangle
$$

where $x_{i, j}$ and $y_{i, j}$ are separated in space by $x_{T}$ and $\theta_{b}$ is the angle of beam $b$. Thus we need to evaluate a fourth moment of log amplitude. The Gaussian assumption provides us with the means; in the Gaussian case the fourth moment is the sum of all possible permutations of products of second moments [Flatté et al., 1979]. Consider first the permutation in which the pair of $u$ from beam $b$ and the pair of $u$ from beam $d$ are kept together. Each pair has an expectation value of $p_{t}$, and the entire contribution of that permutation consists of $p_{1}{ }^{2}$. The other permutations involve second moments with one $u$ from beam $b$ and one $u$ from beam $d$. We consider only the contributions for those pairs of $u$ that are at the same station, since other pairs will have a substantially smaller coherence. It then follows that

$$
\left\langle c^{2}\right\rangle=p_{t}^{2}+\frac{1}{N_{w}^{2}} \sum_{b=1}^{N} \sum_{d=1}^{N} w_{b} w_{d} \frac{\rho_{e}^{2}\left(\theta_{b d}\right)}{M}
$$

and that the variance of $c$ is

$$
\sigma^{2}=\frac{1}{N_{w}^{2}} \sum_{b=1}^{N} \sum_{d=1}^{N} w_{b} w_{d} \frac{\rho_{c}^{2}\left(\theta_{b d}\right)}{M}
$$

The effective number of beams is then determined from (A5), (A9), and (A15) to be

$$
N_{\text {off }}^{-1}=\frac{1}{N_{w}^{2}} \sum_{b=1 d=1}^{N} \sum_{d=1}^{N} w_{b} w_{d} \frac{\rho_{a}^{2}\left(\theta_{b d}\right)}{\left\langle u^{2}\right\rangle^{2}}
$$

and we note that the last ratio is just what we call the normalized ACF. We can use our measurements of the normalized ACF to evaluate $N_{\text {off }}$ for each point on the TCF, and our measurements of the values of $c_{b}$ for the different beams to evaluate the variance of $c_{b}$. Finally, we apply (A9) to find $\sigma$. The above procedure is easily applied to the other two TCFs as well.

\section{ACFs and Their Statistical Errors}

The above principles also apply to the problem of evaluating the ACFs and their errors. In the ACF case the fundamental measurement requires a pair of beams instead of the single beam of the TCF case. In establishing the weights, we ask whether a given pair is independent of another pair. Furthermore, when we form a fourth moment of a quantity, we are dealing with four beams instead of two.

Our procedure for forming the weights is as follows: First, we find all pairs of beams that are separated by a desired angle $\theta$. Then the weight for pair $q$ is given by

$$
w_{q}^{-1}=1+\sum_{p \neq q} p_{a}\left(\theta_{1}\right) p_{a}\left(\theta_{2}\right)
$$

where the sum is over all the other pairs. The angles $\theta_{1}$ and $\theta_{2}$ are chosen so that the product of the $\rho$ 's is the maximum. Again, this form has the correct limiting values.

The expression for the ACF can be given then as

$$
p_{e}(\theta)=\frac{1}{N_{w}} \sum_{p=1}^{N} w_{p} \frac{1}{M} \sum_{s=1}^{N} u_{q s} u_{r s}
$$

where $q$ and $r$ are the indices of the beams making up pair $p$ and $s$ is the index of the station within the array. In evaluating the fourth moment as a product of second moments, we again treat the first permutation completely and then ignore those pairs which belong to different stations in subsequent permutations. We find

$$
N_{\text {off }}^{-1}=\frac{1}{N_{w}^{2}} \sum_{p} \sum_{q} w_{p} w_{q} p_{a n}(\phi) p_{a n}(\psi)
$$

where $p$ and $q$ are different beam pairs with angle difference $\theta$ and $\rho_{a n}$ is the normalized ACF. The angle $\phi$ is the smallest angle obtained by selecting one beam from each of the two beam pairs. The angle $\psi$ is the angle between the other two beams. Again, this is an approximation, which has the right behavior in the limiting case of many beam pairs, each of which is made up of the same beams.

It is of interest to point out that within our approximations, the values of a TCF at different $x_{T}$ and the values of an ACF at different $\theta$ are not correlated.

Acknowledgments. We are grateful for discussions with Zheng-Kang Shen. This work was supported by Defense Advanced Research Projects Agency grant MDA903-86-K-0010.

\section{REFERENCES}

Aki, K. Scattering of P waves under the Montana Lasa, J. Geophys. Res., 78, 1334-1346, 1973.

Aki, $K$., Scattering and attenuation of shear waves in the lithosphere, $J$. Geophys. Res., 85, 6496-6504, 1980.

Aki, K., Source and scattering effects on the spectra of small local earthquakes, Bull. Seismol. Soc. Am., 71, 1687-1700, 1981.

Aki, K. and A.B. Chouet, Origin of coda waves: Source, attenuation and scattering effects, J. Geophys. Res., 80 3322-3342, 1975.

Bergmann, P.G., Propagation of radiation in a medium with random inhomogeneities, Phys. Rev., 70, 486, 1946.

Berteussen, K.A., NORSAR location calibrations and time delay corrections, NORSAR Sci. Rep. 2-73/74, National Technical Information Service, Springfield, Va., 1974.

Berteussen, K.A., $P$ wave amplitude variability at NORSAR, J. Geophys., 41, 595-613, 1975.

Berteussen, K.A., A. Christoffersson, E.S. Husebye and A. Dahle, Wave scattering theory in analysis of $\boldsymbol{P}$ wave anomalies at NORSAR and LASA, Geophys. J.R. Astron. Soc., 42, 403-417, $1975 a$.

Berteussen, K.A., and E.S. Husebye, Amplitude pattem effects on NORSAR $P$ wave detectability, NORSAR Sci. Rep. 1-74/75, National Technical Information Service, Springfield, Va., 1974.

Berteussen, K.A., E.S. Husebye, R.F. Mereu, and A. Ram Quantitative assessment of the crust-upper mantle heterogeneities beneath the Gauribidanur seismic array in southem India, Earth Planet. Sci. Lett., 37. 326-332, 1975b.

Boss, A.P. and I.S. Sacks, Formarion and growth of deep mantle plumes, Geophys. J. R. Attron. Soc., 80, 241-255, 1985.

Capon, J., Characterization of cnust and upper mantle structure under Lasa as a random medium, Bull. Seismol. Soc. Am., 64, 235-266, 1974.

Capon, J., and K.A. Berteussen, A random medium analysis of crust and upper mantle structure under NORSAR, Geophys. Res. Lett., 1, 327-328, 1974. 
Chemov, L.A., Wave Propagation in a Random Medium, MoGraw-Hill, New York, 1960.

Dashen, R., Path integrals for waves in random media, J. Math Phys., 20, 894-920, 1979.

Dashen, R., S.M. Flatte, and S.A. Reynolds, Path-integral treatment of acoustic mutual coherence functions for rays in a sound channel, $J$. Acoust. Soc. Am., 77, 1716-1722, 1985.

Fante, R.L., Electromagnetic beam propagation in turbulent media: an update, Proc. IEEE, 68, 1424-1443, 1980.

Flatte, S.M., Wave propagation through random media: contributions from ocean acoustics, Proc. IEEE, 71, 1267-1294, 1983.

Flatte, S.M. and R.B. Stoughton, Theory of acoustic measurement of intemal-wave strength as a function of depth, horizontal position, and time, J. Geophys. Res., 91, 7709-7720, 1986.

Flatte, S.M., R. Dashen, W.H. Munk, K.M. Watson, and F. Zachariasen, Sound Transmission Through a Fluctuating Ocean, Cambridge University Press, New York, 1979.

Flatté, S.M., S.A. Reynolds, and R. Dashen, Path-integral treatment of intensity and intensity moments for rays in a sound channel, $J$. Acoust. Soc. Am., 82, 967-972, $1987 a$.

Flatté, S.M., S.A. Reynolds, R. Dashen, B. Buehler, and P. Maciejewski, AFAR measurements of intensity and intensity moments, $J$. Acoust. Soc. Am., 82, 973-980, $1987 b$.

Fock, V.A., Theory of radio-wave propagation in an inhomogeneous atmosphere for a raised source, Izv. Akad. Nauk. SSSR, Ser. Fiz., 14, $70,1950$.

Frankel, A., and R.W. Clayton, Finite-difference simulations of seismic scattering: Implications for the propagation of short-period seismic waves and models of crustal inhomogeneity, J. Geophys. Res., 91, 6465-6489, 1986.

Garrett, C., and W.H. Munk, Space-time scales of internal waves: A progress report, J. Geophys. Res., 80, 291-297, 1975.

Given, J.W., and D.V. Helmberger, Upper mantle structure of northwestem Eurasia, J. Geophys. Res., 85, 7183-7194, 1980.

Haddon, R.A.W., and E.S. Husebye, Joint interpretation of $P$ wave time and amplitude anomalies in terms of lithospheric heterogeneities, Geophys.J.R. Astron. Soc., 55, 19-43, 1978.

Husebye, E.S., J. Hovland, A. Christoffersson, K. Astrom, R. Slunga, and C.-E Lund, Tomographical mapping of the lithosphere and asthenosphere beneath southern Scandinavia and adjacent areas, Tectonophysics, 128, 229-250, 1986.

Ishiman, A., Theory and application of wave propagation and scattering in random media, JProc. IEEE, 65, 1030-1061, 1977.

Leontovich, M., and V. Fock, Solution of the problem of propagation of electromagnetic waves along the Earth's surface by the parabolic equation method, J Zh. Eksp. Teor. Fiz., 16, 557-73, 1946.

McLaughlin, K.L., T.W. McElfresh, and R.H. Shumway, Determination of event magnitudes with correlated dats and censoring: A maximum likelihood approach, Geophys. J R. Astron. Soc.,n in press, 1988.
Mintzer, D., Wave propagation in a randomly inhomogeneous medium, J. Acoust. Soc. Am., 25, 922-927, 1953.

Munk, W.H. and F. Zachariasen, Sound propagation through a fluctuating ocean - Theory and observation, J. Acoust. Soc. Am., 59, 818-838, 1976.

Olson, P., G. Schubert, and C. Anderson, Plume formation in the D" layer and the roughness of the core-mantle boundary, Nature, 327, $409-413,1987$.

Powell, C.A., and A.S. Meltzer, Scattering of $\boldsymbol{P}$ waves beneath SCARLET in southem Califomia, Geophys. Res. Lett., 11, 481-484, 1984.

Prokhorov, A.M., F.V. Bunkin, K.S. Gochelashvily, and V.I Shishov, Laser irradiance propagation in turbulent media, Proc. IEEE, 63, 790-811, 1975.

Reynolds, S.A., S.M. Flatté, R. Dashen, B. Buehler, and P. Maciejewski, AFAR measurements of acoustic mutual coherence functions of time and frequency, J. Acoust. Soc. Am., 77, 1723-1731, May 1985.

Sacks, I.S., J.A. Snoke, and E.S. Husebye, Lithosphere thickness beneath the Baluic Shield, Tectonophysics, 56, 101-110, 1979.

Sato, H., Attenuation of $S$ waves in the lithosphere due to scattering by its random velocity structure, J. Geophys. Res., 87, 7779-7785, 1982.

Stoughton, R.B., S.M. Flatte, and B. Howe, Acoustic measurements of internal-wave ms displacernent and ms horizontal current off Bermuda in late 1983, J. Geophys. Res., 91, 7721-7732, 1986.

Strohbehn, J. W. (Ed.), Laser Beam Propagation in the Atmosphere, Springer-Verlag, New York, 1978.

Tatarskii, V.I. The Effects of the Turbulent Atmosphere on Wave Propagation, National Technical Information Service, Springfield, Va., 1971.

Tatarskii, V.I., and V.U. Zavorotnyi, Strong fluctuations in light propagation in a randomly inhomogeneous medium, Progr. Opt., 18 , 204-256, 1980.

Wang, T.I., G.R. Ochs, and S.F. Clifford, A saturation-resistant optical scintillometer to measure $C_{n}{ }^{2}, J$. Opt. Soc. Am., 68, 334-338, 1978.

Wu, R.S., Attenuation of short period seismic waves due to scattering, Geophys. Res. Lett., 9, 9-12, 1982.

Wu, R.S., and K. Aki, The fractal nature of the inhomogeneities in the lithosphere evidenced from seismic wave scattering, Pure Appl. Geophys., 123, 805-818, 1985a.

Wu, R.S., and K. Aki, Elastic wave scattering by a random medium and the small scale inhomogeneities in the lithosphere, J. Geophys. Res., 90, 10261-10273, $1985 b$.

S.M. Flatte and R.-S. Wu, Physics Department, University of Califormia, Santa Cruz, CA 95064.

(Received November 11, 1987;

revised February 22, 1988;

accepted January 24,1988 .) 